\title{
RESEARCH
}

Open Access

\section{An integrative multi-omics approach reveals new central nervous system pathway alterations in Alzheimer's disease}

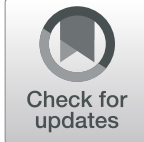

\author{
Christopher Clark', Loïc Dayon ${ }^{2,3,4}$, Mojgan Masoodi ${ }^{2,5}$, Gene L. Bowmann ${ }^{2,6}$ and Julius Popp ${ }^{7,8^{*}}$ (D)
}

\begin{abstract}
Background: Multiple pathophysiological processes have been described in Alzheimer's disease (AD). Their interindividual variations, complex interrelations, and relevance for clinical manifestation and disease progression remain poorly understood. We hypothesize that specific molecular patterns indicating both known and yet unidentified pathway alterations are associated with distinct aspects of AD pathology.

Methods: We performed multi-level cerebrospinal fluid (CSF) omics in a well-characterized cohort of older adults with normal cognition, mild cognitive impairment, and mild dementia. Proteomics, metabolomics, lipidomics, onecarbon metabolism, and neuroinflammation related molecules were analyzed at single-omic level with correlation and regression approaches. Multi-omics factor analysis was used to integrate all biological levels. Identified analytes were used to construct best predictive models of the presence of AD pathology and of cognitive decline with multifactorial regression analysis. Pathway enrichment analysis identified pathway alterations in AD.

Results: Multi-omics integration identified five major dimensions of heterogeneity explaining the variance within the cohort and differentially associated with AD. Further analysis exposed multiple interactions between single 'omics modalities and distinct multi-omics molecular signatures differentially related to amyloid pathology, neuronal injury, and tau hyperphosphorylation. Enrichment pathway analysis revealed overrepresentation of the hemostasis, immune response, and extracellular matrix signaling pathways in association with AD. Finally, combinations of four molecules improved prediction of both AD (protein 14-3-3 zeta/delta, clusterin, interleukin-15, and transgelin-2) and cognitive decline (protein 14-3-3 zeta/delta, clusterin, cholesteryl ester 27:1 16:0 and monocyte chemoattractant protein-1).
\end{abstract}

Conclusions: Applying an integrative multi-omics approach we report novel molecular and pathways alterations associated with AD pathology. These findings are relevant for the development of personalized diagnosis and treatment approaches in AD.

Keywords: Alzheimer's disease, CSF, MOFA, Multi-omics, Biomarkers

\footnotetext{
* Correspondence: julius.popp@pukzh.ch

${ }^{7}$ Old Age Psychiatry, Centre Hospitalier Universitaire Vaudois, Rue du Bugnon 46, 1011 Lausanne, Switzerland

${ }^{8}$ Department of Geriatric Psychiatry, University Hospital of Psychiatry Zürich,

Centre for Gerontopsychiatric Medicine, Minervastrasse 145, P.O. Box 341,

8032 Zürich, Switzerland

Full list of author information is available at the end of the article
}

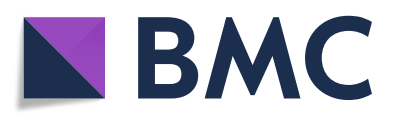

(c) The Author(s). 2021 Open Access This article is licensed under a Creative Commons Attribution 4.0 International License, which permits use, sharing, adaptation, distribution and reproduction in any medium or format, as long as you give appropriate credit to the original author(s) and the source, provide a link to the Creative Commons licence, and indicate if changes were made. The images or other third party material in this article are included in the article's Creative Commons licence, unless indicated otherwise in a credit line to the material. If material is not included in the article's Creative Commons licence and your intended use is not permitted by statutory regulation or exceeds the permitted use, you will need to obtain permission directly from the copyright holder. To view a copy of this licence, visit http://creativecommons.org/licenses/by/4.0/ The Creative Commons Public Domain Dedication waiver (http://creativecommons.org/publicdomain/zero/1.0/) applies to the data made available in this article, unless otherwise stated in a credit line to the data. 


\section{Background}

Along with amyloid pathology and tau-related neurodegeneration, multiple other molecular alterations and pathway dysregulations have been reported in Alzheimer's disease $(\mathrm{AD})$. Indeed, there is strong evidence that pathophysiological changes involving neuroinflammation [1] lipid metabolism [2], one-carbon metabolism [3], amino acids [4], and glucose metabolism [5], among others, are present in AD. However, the contribution and relevance of these alterations to clinical manifestation and progression of the disease as well as their inter-individual variations, and complex interrelations, remain poorly understood. While these processes are generally not considered part of the "core" AD pathology, they may substantially contribute to the development of amyloid pathology and neurodegeneration and precipitate the manifestation of symptoms. As they may be occurring at early clinical and preclinical disease stages, a better understanding of these processes may be highly relevant for both early diagnosis and prognosis and the design of targeted interventions to interfere with developing $\mathrm{AD}$ pathology and clinical disease progression.

'Omics approaches and technologies have made major progress over the past decade to resolve the complexity of the metabolome, lipidome and proteome [6]. As powerful phenotyping technologies, 'omics significantly accelerate the understanding of mechanisms of pathophysiological alterations that underlie complex diseases such as $\mathrm{AD}[7,8]$. Beyond the potential of identifying altered biofluid molecule profiles that could be used as biomarkers, these technological advances also offer the opportunity to explore different types of molecules in parallel by combining multiple 'omics methods. Recent statistical advances have made it possible to integrate the information from multiple data modalities for a thorough exploration of endophenotype networks and biological interactions related to disease [9]. While multi-omics approaches have recently shown their potential in relation to different other pathological conditions [10-12], these methods still need to be more broadly adapted and applied in AD [13].

Here, we hypothesized that specific patterns of proteins, lipids, neuroinflammatory markers, and metabolites are associated with core features of the $\mathrm{AD}$ pathology and indicate disease-related, inter-connected biological pathway alterations. We investigated these alterations across multiple biochemical pathways by using a multi-layer dataset acquired by analysis of cerebrospinal fluid (CSF) from a cohort of elder subjects with normal cognition. In order to integrate data from different 'omics platforms in an unbiased fashion while considering interactions between modalities, we combined different approaches including single 'omics analysis and multi-omics factor analysis (MOFA) [14-16].

\section{Methods}

\section{Study population}

One hundred and twenty community dwelling individuals, aged 55 or older, including subjects with normal cognition, mild cognitive impairment (MCI), or mild $\mathrm{AD}$ dementia (defined as previously described [3]), were enrolled into a brain aging study conducted in the Department of Psychiatry and the Department of Clinical Neurosciences, University Hospital of Lausanne, Switzerland. They were recruited among memory clinic outpatients or through advertisement. An overall clinical, neurological, and comprehensive neuropsychological assessment was performed between 2013 and 2016, which included the Mini Mental State Examination (MMSE, [17]) and Clinical Dementia Rating (CDR, [18]). Candidates with unstable medical conditions or with neurological or psychiatric diseases that could interfere with cognitive performance were excluded as previously described [19]. Clinical and neuropsychological follow-up evaluations were performed at 18 and 36 months using the same methods and tests.

\section{Study procedures \\ Clinical assessment}

We determined Mini-Mental State Examination (MMSE), CDR, and CDR sum of boxes (CDR-SoB), for all participants. CDR-SoB and CDR were based on the information available from the participant and his/her relative, the clinical examination, and comprehensive neuropsychological test performance, as previously described [19].

\section{Biochemical sample collection and handling}

Ten to $12 \mathrm{ml}$ of cerebrospinal fluid (CSF) obtained from lumbar punctures conducted after an overnight fast at participant inclusion were spun down at $4{ }^{\circ} \mathrm{C}$, immediately aliquoted, and snap frozen at $-80{ }^{\circ} \mathrm{C}$ until assayed [19], with no freeze-thaw cycles allowed. Samples were stored for a maximum of 3 years before analysis. Study personnel blinded to clinical data performed biochemical and genetic analyses.

\section{Cerebrospinal fluid $A D$ biomarkers}

CSF beta-amyloid 1-42 (A $\left.\beta_{1-42}\right)$, total-tau (Tau), and tau phosphorylated at threonine 181 (P-Tau) concentrations were measured using commercially available ELISA kits (Fujirebio, Gent, Belgium) in all samples within the cohort.

\section{Analyte measurements}

Multiple 'omics data from different pathways and various biological levels were acquired from a vast majority of participants within the cohort $(n=114 / 120$ for proteomics, 118 for metabolomics, 119 for neuroinflammation and one-carbon metabolism, and 120 for lipidomics). 
CSF samples were measured using an untargeted shotgun proteomic workflow based on liquid chromatography (LC) tandem MS (MS/MS) using an Ultimate 3000 RSLC nano system and a hybrid linear ion trapOrbitrap (LTQ-OT) Elite (Thermo Scientific, San Jose, CA, USA) [20, 21]. Relative quantification of proteins between the samples was obtained using isobaric tagging with the tandem mass tag technology [22]. Full experimental details and parameters of the proteomic analysis have been published previously [23, 24]. A targeted subset of thirty-seven CSF inflammatory proteins including IFN $\gamma$, IL-1 $\beta$, IL-2, IL-4, IL-6, IL-8, IL-10, IL-13, TNF $\alpha$, IL-1 $\alpha$, IL-5, IL-7, IL-12/23p40, IL-15, IL-16, IL-17A, TNF $\beta$, VEGFA, Eotaxin, MIP-1 $\beta$, Eotaxin-3, TARC, IP10, MIP-1 $\alpha$, MCP-1, MDC, MCP-4, VEGF-C, VEGF-D, Tie-2, sFLT-1 (VEGFR-1), PIGF-1R, bFGF, SAA1, CRP, sVCAM-1, and sICAM-1 were separately quantified using a sandwich immunoassay (Meso Scale Diagnostics (MSD), Rockville, MD, USA), according to the manufacturer's instructions. This platform has been validated by the manufacturer (https://www.mesoscale.com/ /media/ files/product\%20inserts/neuroinflammation $\% 20$ panel\%2 01\%20human\%20insert.pdf) and has been previously used successfully [25].

CSF lipids were quantified using an MS-based shotgun approach [26]. This technology can cover 22 quantifiable different lipid classes encompassing more than 200 lipid species; it achieves absolute quantification, by inclusion of internal standards for every lipid class measured. Figure-of-merits are an average coefficient of variation of $<10 \%$ (intra-day), approximatively $10 \%$ (inter-day), and approximatively $15 \%$ (inter-site) for most lipid species.

Metabolomic profiling was carried out by means of ${ }^{1} \mathrm{H}$ NMR spectroscopy, as reported previously [27]. This approach covered major metabolic pathways, including amino acids, carboxylic acids, and central energy metabolism. Metabolites within the one-carbon pathway are a hypothesis-driven subset of metabolites [3] and were separately analyzed using LC-MS/MS as previously described [28] with an Accela UHPLC 1250 Pump coupled to a TSQ Quantum Vantage triple quadrupole mass spectrometer equipped with a heated electrospray ionization source (Thermo Scientific). Selected reaction monitoring transitions have been described previously [28].

The initial number of analytes measured in CSF, the final number of analytes selected per platform (a total of 891 analytes covered), and quantification method used for each platform are summarized in Table 1.

\section{Genetic measures}

The APOE genotype was determined by PCR as previously described [29]. Participants with one or more e4 alleles were classified as carriers.

\section{Data preparation and transformation Lipidomics}

Twenty-six high-quality intact lipids with less than $5 \%$ of null values were selected as continuous numerical markers from 65 original measurements. Numerical lipid marker values were log10-transformed prior to analysis.

\section{Metabolomics}

Seventy-one peak integrals were originally measured in CSF. Sixty-three analytes with less than $5 \%$ missing values were selected from the obtained spectra. Peak integral values were $\log 10$-transformed prior to analysis.

\section{One-carbon metabolomics}

Seventeen analytes were initially measured in CSF. Some analytes could not be measured in the majority of samples and were excluded from the analysis (i.e., homocysteic acid, dimethylglycine, betaine, total homocysteine, pyridoxine, and pyridoxamine); taurine and glycine data were inconsistent and were also filtered out resulting in 9 measured analytes (i.e., choline, cystathionine, methionine, riboflavin, S-adenosylhomocysteine, Sadenosylmethionine, serine, cysteine and 5methyltetrahydrofolate). Analytes with more than 5\% missing data points were also removed. Numerical values were $\log 10$-transformed prior to analysis.

Table 1 Datasets used in this study

\begin{tabular}{llll}
\hline Dataset & Analytes initial/final & Quantification technique & References \\
\hline Proteomics & $791 / 768$ & LC-MS/MS & {$[23,24]$} \\
Neuroinflammation & $38 / 21$ & Multi-array sandwich immunoassay & {$[29-31]$} \\
One-carbon metabolism & $17 / 9$ & LC-MS/MS & {$[28,32]$} \\
Metabolomics & $71 / 63$ & ${ }^{1}$ H NMR & {$[27]$} \\
Lipidomics & $65 / 26$ & MS & [26, 33] \\
Biomarkers of core AD pathology & $3 / 3$ & ELISA & {$[30]$} \\
\hline
\end{tabular}

Available datasets from the cohort along with the number of analytes measured in this study and the associated quantification methods. For each dataset the initial number of analytes quantified, the number of measurements remaining after quality control, quantification technique used, and technical references are indicated. LC-MS/MS, liquid chromatography tandem mass spectrometry; ${ }^{1} \mathrm{H}$ NMR, proton nuclear magnetic resonance; MS, mass spectrometry; ELISA, enzymelinked immunosorbent assay 


\section{Neuroinflammatory markers}

Thirty-eight markers were measured in CSF. Calibration curves, batch effects, and analytes with more than 5\% missing data points were removed, and lower limit of quantification was controlled. After this quality control, 17 markers were removed, resulting in 21 markers selected. Concentrations were $\log 10$-transformed prior to analysis.

\section{Proteomics}

Relative quantification data were available for all subject samples as $\log 2$ ratios as previously described [24]. Analytes with more than $5 \%$ missing data points were removed, resulting in 768 proteins measured from an initial number of 791 .

Before analysis, outliers (i.e., data points that exceeded the cutoff value of mean $\pm 3 \times$ standard deviation) were replaced by the cutoff value in all datasets $(n=28$ for lipidomics, 36 for metabolomics, none for one-carbon metabolomics, 17 for neuroinflammatory markers, and 345 for proteomics). For all datasets, this represented below $1 \%$ of all data points. Missing values were replaced using an iterative Markov chain Monte Carlo method before single-modality feature selection approaches, but were not replaced for multi-omics analysis as the MOFA method can handle large proportions of missing values [14].

\section{Statistical and analytical approaches}

Descriptive statistics for the cohort were performed using $t$ tests comparing $\mathrm{AD}$ and control groups for continuous variables and chi-square tests for categorical variables. Data was clustered by hierarchical clustering across samples and factors values or loadings.

\section{Feature selection methods}

Single-modality approaches To overcome the bias resulting from correlation between variables and thus unreliability and saturation of standard regression techniques, we used Elastic-Net regularization $(\alpha=0.5)$ for regression analysis. This was performed separately for each individual 'omics platform in the whole cohort using R software with custom routines implementing the glmnet package [34]. Each pre-specified CSF biomarker endpoint was considered as a continuous dependent variable and associated features were identified using a value of $\lambda$ (lambda) that minimized the 10 -fold cross validated error.

Multi-omics factor analysis This analysis was performed using the MOFA package on the whole cohort in $\mathrm{R}$ and Python software [14]. Latent factors (also referred as LFs) were selected to explain a minimum of $2 \%$ variance in at least one data type. The MOFA model was trained over 938 iterations with a convergence threshold of 0.1. Individual analytes were selected if their normalized absolute loading value was $>0.8$ within any given LF in order to include only analytes with strong associations. More details about the MOFA method can be found in Additional file 1. The trained MOFA model was validated using both a correlation approach and CSF AD biomarker predictions (Additional file 2, Figures S1 and S2, respectively).

Associations with CSF biomarkers of AD In order to evaluate the correlations of the analytes identified by the MOFA model with CSF biomarkers of AD (CSF A $\beta_{1-42}$, Tau and P-Tau), we used two-tailed Spearman's rho. Benjamini-Hochberg correction of $P$ value for multiple testing was then applied using a false-discovery rate of 0.1 .

Models for the prediction of $\mathrm{AD}$ and of cognitive decline Predictions were ran using the $g l m$ package in R. Subjects were classified as controls or AD, according to the presence or absence of an AD CSF biomarker profile, defined by a CSF $\mathrm{P}-\mathrm{Tau} / \mathrm{A} \beta_{1-42}$ ratio $>0.0779$ based on center data [30]. We constructed a reference model including the following covariates for AD prediction: age, sex, years of education, baseline MMSE score, and APOE4 carrier status. MMSE change at last available follow-up for one hundred and three participants, with nineteen participants followed up at 18 months only, and eighty-four at 36 months, was used to classify participants with decreased global cognition as follows: MMSE score at baseline - MMSE at last follow-up visit $\geq 2$. Another reference model including age, sex, years of education, baseline MMSE score, APOE4 carrier status, and time to last follow-up was constructed for cognitive decline prediction. We then used an iterative approach, first adding all analytes identified by the MOFA model individually to the above reference model and selected the model displaying the smallest Akaike information criterion (AIC) value to select the best molecule to add at each iteration. We repeated this process over successive iterations, adding a single analyte each time. Performance of the models was analyzed by comparing area under the curve (AUC) of the resulting ROC curves using the DeLong method. No further improvements to the AUC were observed after five iterations for both predictions. Confusion matrices to assess sensitivity and specificity were calculated for all models.

\section{Pathway enrichment}

Proteins selected by the MOFA model were searched for in the UniProt database [35], and their entry number was then subsequently used within the Reactome 
database [36]. A separate over-representation analysis was performed for each LF. This analysis used hypergeometric distribution to determine which pathways and biological reactions were over-represented within the dataset. Over-represented pathways were then manually grouped into broader ontology-based categories (Additional file 3, Table S1).

\section{Results}

\section{Cohort description}

The clinical and demographical characteristics of the participants included in this study are shown in Table 2.

\section{Single-modality feature selection}

Elastic-Net regression within each single 'omics modality identified 82 molecules associated with CSF "core" biomarkers of AD pathology (i.e., $\mathrm{A} \beta_{1-42}$, Tau and P-Tau) within the whole cohort (Tables 3,4 , and 5). Twenty out of the thirty-seven proteins selected were correlated with at least one CSF AD biomarker. Only two neuroinflammatory molecules displayed no correlation with CSF AD biomarkers. Conversely, only two molecules at metabolomics level and two lipids were correlated with CSF AD biomarkers. Finally, total cysteine showed no correlations (Additional file 3, Table S2). Strikingly, distinct panels of CSF analytes were associated with either $\mathrm{A} \beta_{1-42}$, or Tau and P-Tau, reflecting alterations of different pathways in relation to amyloid pathology, neurodegeneration, and tau pathology, with very little overlap (Fig. 1). Only protein 14-3-3 zeta/delta was associated with all three biomarkers.

\section{Overview of the MOFA model}

In parallel, we trained a MOFA model on the whole cohort, to identify major dimensions of heterogeneity (latent factors; LFs) responsible for the variance within the cohort. This model identified five LFs that each explained a minimum of $2 \%$ variance in at least one of the analyzed metabolic levels. Among these factors, LF1 and LF2 were present in most multi-omics modalities, revealing a broad participation to variance within the
Table 3 Analytes associated with CSF $A \beta_{1-42}$

\begin{tabular}{ll}
\hline & Coeff. \\
\hline $\begin{array}{ll}\text { Neuroinflammation } \\
\text { C-reactive protein }\end{array}$ & 17.9542 \\
Monocyte chemoattractant protein-1 & -78.6254 \\
Proteomics & \\
Spermine synthase & 109.3853 \\
WAP four-disulfide core domain protein 2 & 93.78915 \\
Ephrin-B2 & 72.52614 \\
Neuroendocrine convertase 2 & 55.25737 \\
WAP four-disulfide core domain protein 1 & 42.96994 \\
Spectrin beta chain, non-erythrocytic 5 & 37.99513 \\
Neuropentraxin2 & 37.29243 \\
Chondroadherin & 32.78591 \\
Reelin & 19.927 \\
Sodium/potassium-transporting ATPase subunit alpha-2 & 17.62631 \\
von Willebrand factor & 17.59776 \\
Mast/stem cell growth factor receptor kit & 17.49956 \\
Lymphatic vessel endothelial hyaluronic acid receptor 1 & 7.896977 \\
Neurotrimin & 1.179857 \\
Acid ceramidase & 0.892224 \\
Protein shisa-6 & 0.412964 \\
Monocyte chemoattractant protein 1 & -12.5945 \\
14-3-3 protein zeta/delta & -186.61 \\
\hline A & -230.535 \\
\hline
\end{tabular}

Analytes within the whole cohort with a significant association with CSF $A \beta_{1-42}$ sorted by decreasing absolute association strength within each modality. For each analyte, the coefficient obtained by Elastic-Net regression is shown

cohort (Fig. 2). On the other hand, the remaining LFs only captured variance across some modalities (three for LF4 and LF5, two for LF3) and had a smaller contribution to overall variance. Across all LFs, the CSF AD biomarkers accounted for $38.5 \%$, proteins $39.8 \%$, lipids $10.3 \%$, neuroinflammation markers $10.3 \%$, one-carbon metabolites $9 \%$, and other metabolites $3.7 \%$ of the variance contained within the cohort (Fig. 2). We next produced clustered heatmaps of the weight (i.e., the

Table 2 Study cohort

\begin{tabular}{|c|c|c|c|c|}
\hline & Whole cohort $(n=120)$ & Control $(n=79)$ & $\mathrm{AD}(n=41)$ & $P$ value \\
\hline Age (years) & $70.37 \pm 7.92$ & $68.42 \pm 8.23$ & $74.15 \pm 5.7$ & $<0.001$ \\
\hline Sex (\%, female) & 64.2 & 67.1 & 58.5 & 0.354 \\
\hline Education (years) & $12.37 \pm 2.7$ & $12.51 \pm 2.7$ & $12.10 \pm 12.1$ & 0.404 \\
\hline CDR-SoB & $1.054 \pm 1.6$ & $0.456 \pm 0.9$ & $2.20 \pm 2.0$ & $<0.001$ \\
\hline MMSE & $26.94 \pm 3.08$ & $27.85 \pm 2.28$ & $25.15 \pm 3.71$ & $<0.001$ \\
\hline P-Tau/A $\beta_{1-42}$ ratio & $0.088 \pm 0.082$ & $0.048 \pm 0.127$ & $0.165 \pm 0.104$ & $<0.001$ \\
\hline APOEع4 carrier (\%) & 29.6 & 17.7 & 56.1 & $<0.001$ \\
\hline
\end{tabular}

Characteristics of the study cohort. Mean values \pm standard deviation are presented. Per definition, participants within the AD group all presented a positive AD CSF biomarker profile, defined by a $\mathrm{P}$-tau/A $\beta_{1-42}$ ratio $>0.0779$. $P$ value was obtained from $t$ test for continuous variables or chi-square statistics for sex 
Table 4 Analytes associated with CSF Tau

\begin{tabular}{|c|c|}
\hline & Coeff. \\
\hline \multicolumn{2}{|l|}{ Lipidomics } \\
\hline DAG 34:0 & 1080.894 \\
\hline PC 32:0 & 482.8502 \\
\hline PC 34:1 & 357.532 \\
\hline LPA 16:0 & 117.3799 \\
\hline SE 27:1 18:3 & -15.4237 \\
\hline SE $27: 1 \quad 18: 2$ & -22.0295 \\
\hline \multicolumn{2}{|l|}{ Metabolomics } \\
\hline Glycoproteins & 519.5138 \\
\hline 3-Hydroxyisovaleric acid & 154.6212 \\
\hline Hydroxybutyric acid & 71.2998 \\
\hline S56 & -5.1297 \\
\hline S62 & -49.1323 \\
\hline Glucose & -134.352 \\
\hline \multicolumn{2}{|l|}{ One-carbon metabolism } \\
\hline S-adenosylhomocysteine & 264.4874 \\
\hline Choline & 15.9208 \\
\hline 5-Methyltetrahydrofolate & -361.534 \\
\hline \multicolumn{2}{|l|}{ Neuroinflammation } \\
\hline Soluble fms-like tyrosine kinase 1 & 473.9178 \\
\hline$\|-15$ & 173.1296 \\
\hline Soluble vascular cell adhesion molecule-1 & 159.6389 \\
\hline Soluble intracellular cell adhesion molecule-1 & 90.9487 \\
\hline Monocyte chemoattractant protein-1 & 41.9127 \\
\hline
\end{tabular}

\section{Proteomics}

14-3-3 protein zeta/delta

SPARC related modular calcium binding protein 1

Fructose-biphosphate aldolase A

Chondroadherin

Dynein heavy chain 10, axonemal

$-11.34$

Glia-derived nexin

$-13.09$

A-kinase anchor protein 11

$-18.52$

Reelin

Augurin

$-43.98$

Spectrin beta chain, non-erythrocytic 1

$-52.34$

Sialate O-acetylesterase

$-56.71$

Proline-rich acidic protein 1

$-80.54$

Fibromodulin

$-84.41$

Cathepsin D

$-144.10$ 
Table 4 Analytes associated with CSF Tau (Continued)

\begin{tabular}{ll}
\hline & Coeff. \\
\hline Insulin-like growth factor-binding protein 7 & -169.27 \\
Ectonucleotide pyrophosphatase/phosphodiesterase family member 2 & -213.32
\end{tabular}

Analytes within the whole cohort with a significant association with CSF Tau sorted by decreasing absolute association strength within each modality. For each analyte, the coefficient obtained by Elastic-Net regression is shown. S56 and S62 represent different unidentified metabolites

association of an individual molecule with the LF) of each analyte across different LFs (Fig. 3a-e) revealing specific patterns of associations between analytes within each analyzed multi-omic level and LFs. For example, a subset of proteins with a negative association with LF1 have a positive association with LF2 (Fig. 3a) and molecules within the one-carbon metabolism are differentially associated with LF1 and LF2 (Fig. 3c). These patterns suggest groups of molecules interacting together with specific effects on LFs through common pathways. Because only three CSF core AD biomarkers were measured, we did not produce heatmaps to analyze the association of $A \beta_{1-42}$, Tau and P-Tau with these five LFs, but rather, we inspected their absolute individual

Table 5 Analytes associated with CSF P-Tau

\begin{tabular}{ll} 
& \\
\hline Lipidomics & Coeff. \\
PC 34:1 & 75.7244 \\
PC 32:0 & 41.1825 \\
DAG 34:0 & 32.4248 \\
SE 27:1 18:1 & 9.313 \\
LPE 22:6 & 1.2951 \\
SE 27:1 18:2 & -13.6849 \\
Metabolomics & \\
Glycoproteins & 42.4426 \\
3-Hydroxyisovaleric acid & 15.143 \\
S61 & -0.3484 \\
S59 & -1.5514 \\
S56 & -15.8893
\end{tabular}

\section{One-carbon metabolism}

S-adenosylhomocysteine

5-Methyltetrahydrofolate

\section{Proteomics}

SPARC-related modular calcium binding 1

Brain abundant membrane attached signal protein 1

4.028155

Neuromodulin

1.626098

Thymosin beta-10

1.291504

14-3-3 protein zeta/delta

1.10139

Pyruvate kinase PKM

0.189499

Analytes within the whole cohort with a significant association with CSF P-Tau sorted by decreasing absolute association strength within each modality. For each analyte, the coefficient obtained by Elastic-Net regression is shown. $\mathbf{S 5 6}$ S59 and S61 represent different unidentified metabolites loadings across all LFs (Fig. 4). This revealed that individual CSF AD biomarkers had different contributions across the identified LFs. CSF Tau and P-Tau levels were strongly associated with LF1, LF2, and LF3, while $A \beta_{1-42}$ was the main contributor to variance among the CSF AD biomarkers to LF4 and LF5 indicating that these latter LFs were associated with amyloid pathology and the former with tau pathology and neurodegeneration.

\section{Individual analyte contributions to LFs}

We next addressed the contribution of individual molecules to variance within the cohort and how these molecules aligned with CSF AD biomarkers. We selected analytes with absolute normalized loadings $>0.8$ within any given LF derived from the MOFA model. This approach selected 37 proteins, 7 neuroinflammatory markers, 3 one-carbon metabolites, 5 lipids, and 7 other metabolites (not counting analytes selected in multiple LFs) that contributed the most to variance within the cohort (Table 6). We next investigated the relationship between the identified analytes and the expression levels of CSF AD biomarkers in the relevant LFs. We have shown that the LFs are differentially associated with the individual CSF AD biomarkers. Molecules selected within each LF are therefore associated with the CSF AD biomarkers. Indeed, twenty out of thirty-seven proteins were correlated with at least one CSF AD biomarker. Only two neuroinflammatory molecules displayed no correlation with CSF AD biomarkers. Conversely, only two molecules at metabolomics level and two lipids were correlated with CSF AD biomarkers. Finally, in one-carbon metabolism, total cysteine showed no correlations (Additional file 3, Table S2). As they were selected by the MOFA model, these molecules are part of LFs who have an effect on the variance within the cohort. By considering which LF they were selected from, we can infer that they are part of an interacting set of analytes who is associated with changes in CSF AD biomarkers. For example, despite showing no correlations with any CSF $\mathrm{AD}$ biomarker, total cysteine is selected by our MOFA model in LF1, LF2, and LF5. Because these LFs have strong associations with Tau, P-Tau, and $\mathrm{A} \beta_{1-42}$ (Fig. 4), total cysteine is related to a pathway or group of interacting molecules in these LFs that together are associated with the markers of core AD pathology. Kininogen1 (KNG1) also displayed no correlation with CSF AD biomarker, but we can infer it is part of a group of 


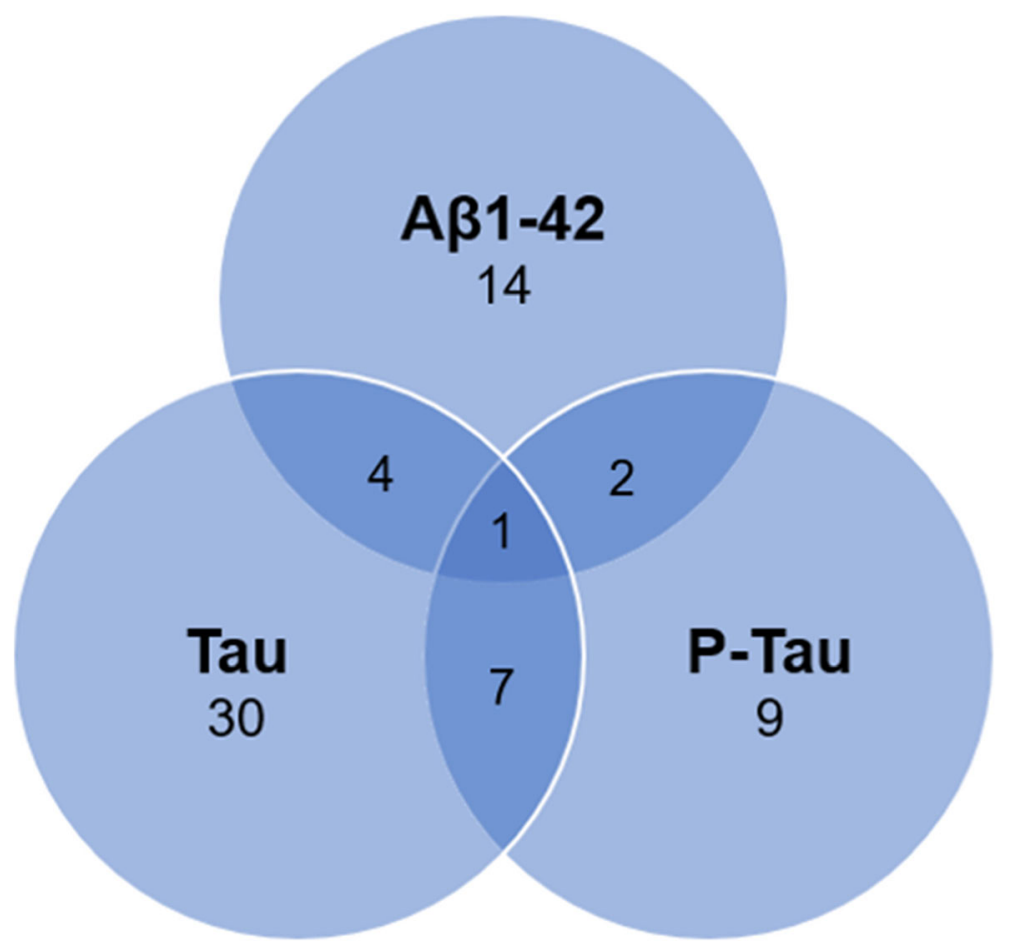

Fig. 1 Venn diagram of associations with CSF core AD biomarkers. Venn diagram of associations of analytes obtained by regression models with CSF core AD biomarkers. Number of molecules identified as well as those shared between biomarkers is shown. The full list of associated molecules is presented in Tables 3, 4, and 5

molecules associated with Tau and P-Tau, since it was selected in LF2. In some cases, the MOFA model revealed supplementary associations. For example, cholesteryl ester SE 27:1 16:0 showed no correlations with $\mathrm{A} \beta_{1-42}$ and strong correlations with both Tau and PTau. This lipid was selected by our MOFA model in LF4, however, suggesting it is part of an interactome with a strong association with $A \beta_{1-42}$.

\section{Cross-modality interactions}

Some of the identified LFs only contain a subset of the tested modalities (Fig. 2). For instance, one-carbon metabolism and metabolomics were only weakly associated with LF3 and LF4, whereas lipidomics was nearly absent from LF3 and LF5. Therefore, the contribution of individual LFs to total variance results from a specific combination of the different 'omics modalities. In addition, individual molecules also presented different patterns of association across LFs. For example, a subset of lipids, including PC 32:0, PC 34:1, LPA 18:3, and TAG 54:3, had a strong positive association with LF2 and a weak negative association with LF4. Since LF2 was associated with all tested modalities (Fig. 2), this indicates these analytes interact within multiple biological pathways and could be within a hub of metabolic changes. Furthermore, LF2 is associated with both Tau and P-Tau indicating neurodegeneration and tau pathology could therefore relate to a more general metabolic alteration. This is supported by the association of PC 32:0 with tau pathology in single 'omics. In contrast, LF4 is strongly associated with amyloid pathology and it is only associated with changes in lipids and proteins (in addition to CSF AD biomarkers). Therefore, only a subset of lipids directly interacts with amyloid pathology. Taken together, these results show that the different aspects of $\mathrm{AD}$ pathology derive from fundamentally different biological pathways and alterations.

\section{Prediction of $A D$ pathology and cognitive decline using MOFA-selected molecules}

In addition to associations with CSF biomarkers of $\mathrm{AD}$ pathology, we found associations with $\mathrm{AD}$ reported in the literature for 37 out of 58 metabolites selected by our MOFA model (Table 6). Also, 29 of the selected proteins and 5 lipids correlated with either baseline CDR-SoB score or MMSE score Additional file 3, Table S3), while,14 of the selected proteins were associated with the presence of cognitive impairment at baseline in regression models (Additional file 3, Table S4). Therefore, in order to confirm a posteriori the importance of the molecules with high weights selected from LFs within the MOFA model, we evaluated their ability to predict either cerebral AD pathology or global cognitive decline. The model for AD prediction selected four 


\section{A Variance explained per modality}

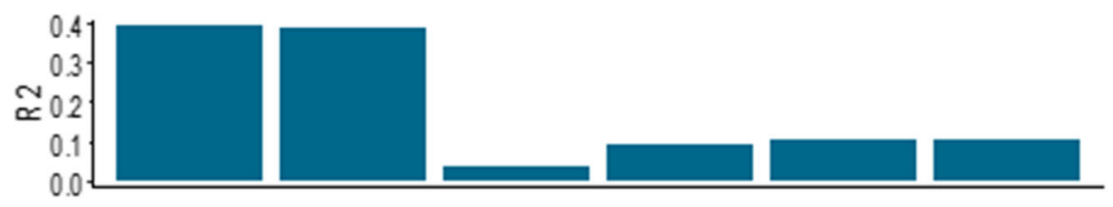

B Variance explained per factor
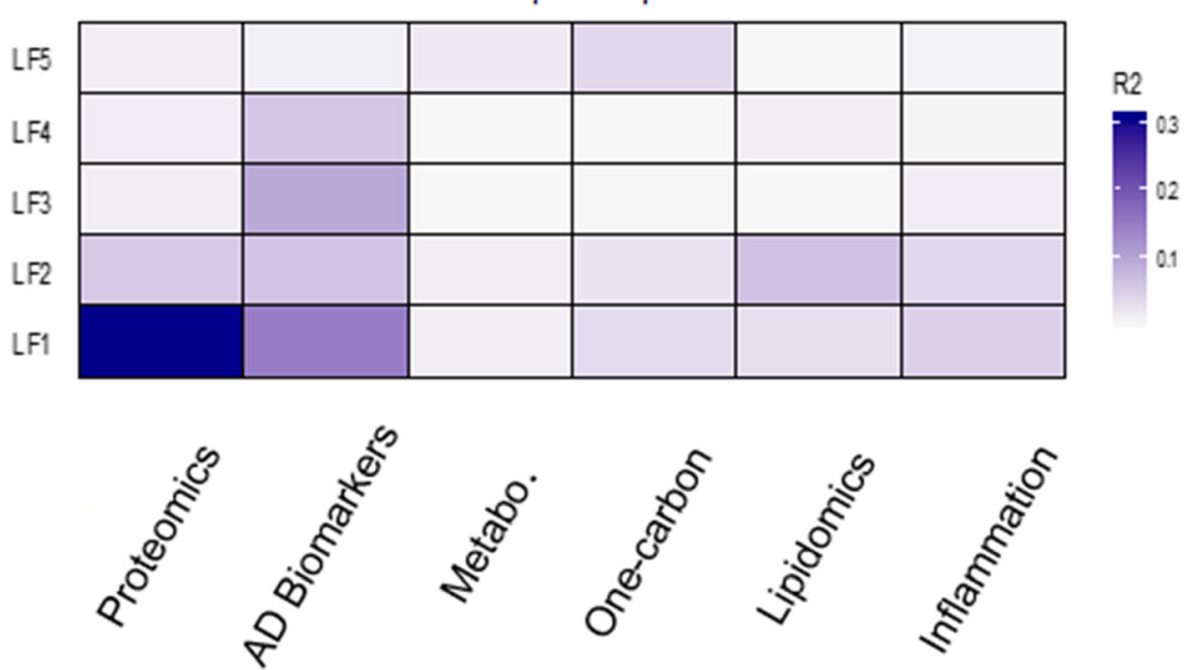

Fig. 2 Overview of the MOFA model. Overview of the trained MOFA model showing variance (R2) within the cohort explained by each modality (top) and latent factors (LFs, bottom) from the trained MOFA model

analytes: protein 14-3-3 zeta/delta, clusterin, interleukin15 , and transgelin-2, that together improved the AUC of the ROC curve when compared to the reference model (Fig. $5 \mathrm{a}, p$ value $=0.002$ ). In addition, both sensitivity (0.71 to 0.86$)$ and specificity ( 0.87 to 0.96$)$ were improved from the reference model. Further, adding to a reference model for the prediction of cognitive decline, four selected molecules, protein 14-3-3 zeta/delta, clusterin, cholesteryl ester 27:1 16:0, and monocyte chemoattractant protein-1, improved its AUC (Fig. 5b, $p$ value $=0.0047)$. This also improved sensitivity ( 0.56 to 0.80 ) but not specificity ( 0.89 to 0.88$)$. For both prediction of cerebral $\mathrm{AD}$ and of cognitive decline, the addition of single molecules to the reference models did not improve prediction (data not shown).

\section{Metabolic pathway enrichment}

Using the Reactome database and coarse-grain ontological categories (See the "Methods" section and Additional file 3, Table S1), we investigated which biological pathways were over-represented within each LF for the proteomic modality. Other modalities were not analyzed in this fashion since they were selected a priori to represent distinct metabolic pathways (one-carbon metabolism and inflammatory markers) or did not contain enough molecules to conduct pathway analysis. Lipids were also excluded from this analysis since our quantification method did not allow to dissociate between different isoforms of compounds with the same chemical formula. This approach revealed an overrepresentation of the hemostasis (28.8\%), immune response (20.8\%), and extracellular matrix signaling pathways (8.8\%) (Fig. 6).

\section{Discussion}

Here, we applied a multi-layered integrative approach to disentangle sources of variance within a cohort of elderly participants with normal cognition, mild cognitive impairment, or mild $\mathrm{AD}$ dementia. We identified five major dimensions of heterogeneity that together comprehensively explained the variance within the cohort and were associated with core AD pathology. Further analysis revealed multiple interactions between single 'omics modalities, distinct multi-omics molecular 


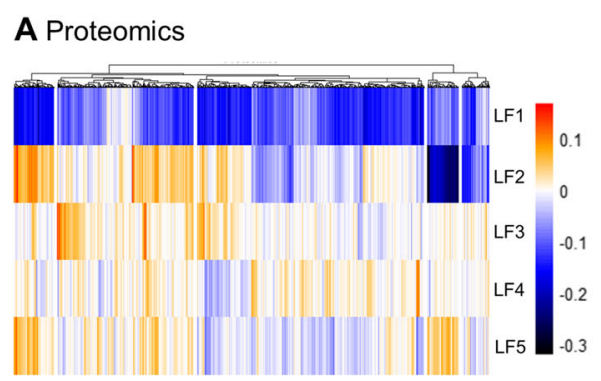

B Neuroinflammation

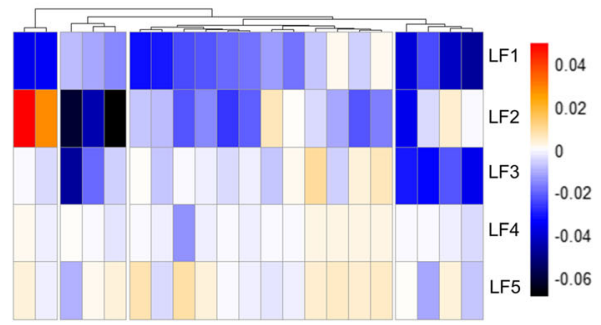

D Metabolomics

C One-carbon
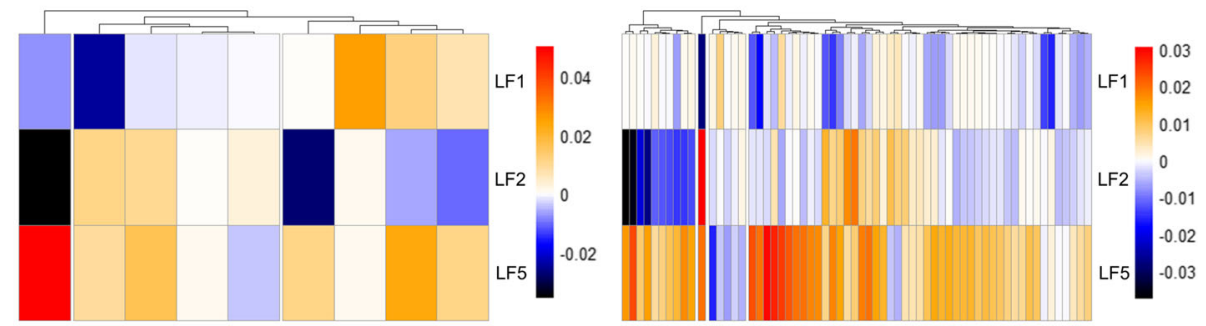

E Lipidomics

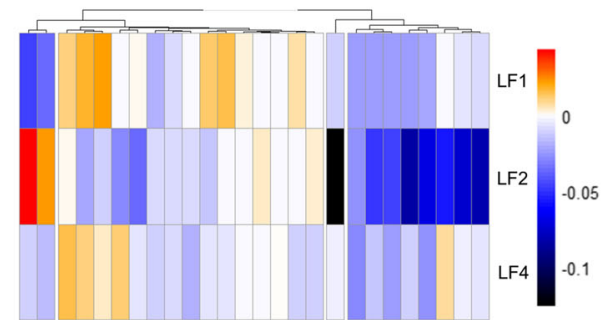

Fig. 3 Clustering of loadings across latent factors. Heatmaps of hierarchical clustering of the measured loadings across in LFs for data obtained from proteomics (a), neuroinflammation markers (b), one-carbon metabolism (c), metabolomics (d), and lipidomics (e) showing clusters of analytes along the $X$-axis and the association of each individual analyte with each LF (shown on the $Y$-axis). Note the distinct pattern within each LF. Color scale indicates both the direction and strength of relative associations

patterns differentially associated with amyloid aggregation, neurodegeneration, and tau hyperphosphorylation, and novel molecules associated with cognitive impairment. Specific signatures of four molecules improved the accuracy of both $\mathrm{AD}$ and cognitive decline prediction. Additionally, pathway enrichment showed overrepresentation of the hemostasis, immune response and extracellular matrix signaling pathways in association with AD.

\section{Single modality feature selection}

We first used Elastic-net regression, to identify molecules associated with individual biomarkers of CSF AD pathology without considering any possible interactions between different 'omics modalities. This approach identified several proteins (SPARC-related modular calciumbinding protein 1 , brain acid soluble protein 1 , neuromodulin, pyruvate kinase PKM, thymosin beta-10, 14-33 protein zeta/delta, and fructose-bisphosphate aldolase A) in strong accordance with recent studies of the $A D$
CSF proteome $[24,65]$. The zeta/delta isoform of protein 14-3-3 was associated with $A \beta_{1-42}$, Tau, and P-Tau levels. This apoptosis inhibitor, one of the most abundant proteins in the brain, was previously found to exhibit altered levels in $\mathrm{AD}$ and modulate AD risk [66, 67]. We also identified associations of neurofilament medium polypeptide with Tau levels and of reelin with $A \beta_{1-42}$ and Tau levels. Both these molecules have previously been associated with AD [68-70]. Regarding neuroinflammatory molecules, $\mathrm{C}$-reactive protein and monocyte chemoattractant protein-1 have previously been associated with $\mathrm{AD}$, albeit in plasma [71]. In addition, we have also previously shown that soluble intracellular cell adhesion molecule- 1 in CSF is associated with AD [30]. At metabolite level, we identified 10 molecules in CSF associated with Tau and P-Tau, which differ from the blood biomarkers associated with $\mathrm{AD}$ identified in a recent study in a large sample [72]. Overall, our approach identified more molecules associated with $\mathrm{AD}$ pathology as compared to previous studies. A likely source of 


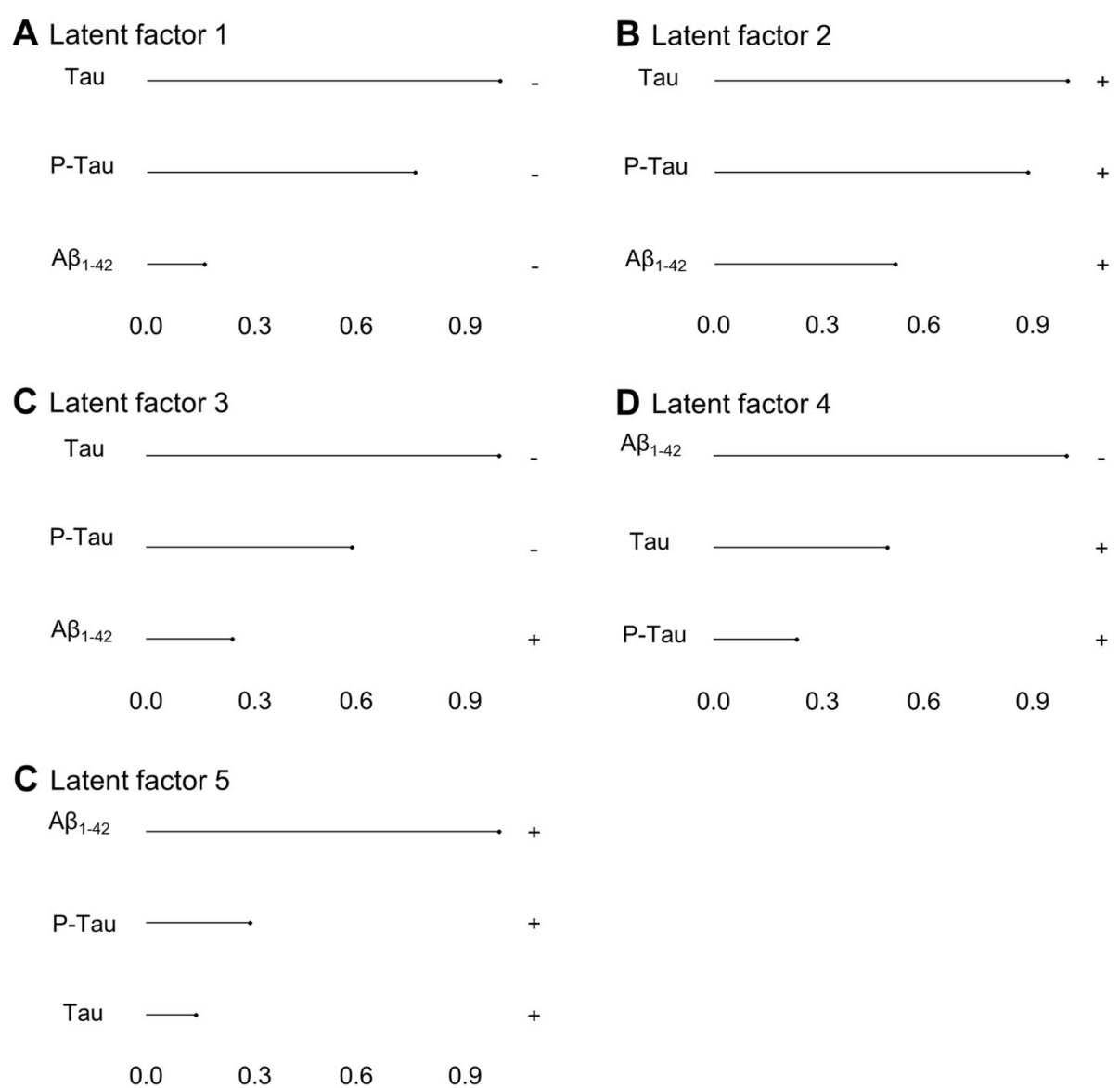

Fig. 4 Loadings of CSF AD biomarkers. Normalized loadings of CSF AD biomarkers shown on the $X$-axis across the five latent factors of the trained MOFA model. Positive or negative signs indicate the relative direction of the CSF AD biomarkers with the associated latent factor. Note that signs are relative within a single latent factor for biomarker weights

differences is the use of Elastic-Net regression in the current study which eliminates saturation of the regression and could therefore identify more associations. On the other hand, our approach did not identify any lipids or metabolites associated with $A \beta_{1-42}$ levels. It is possible that it is not the individual levels of these molecules that are associated with $A \beta_{1-42}$ levels, but rather lipidomic pathway alterations where associations of individual lipids are weak but the overall pathway in which they are embedded is strongly associated with amyloid pathology.

\section{Heterogeneity within the cohort}

An important strength of our study is to consider all interactions between multiple biological levels and their associations with the heterogeneity within the cohort. This was achieved by training a MOFA model on the multi-omics dataset which has the advantage of not giving any additional analysis weight to the established CSF biomarkers of core $\mathrm{AD}$ pathology while also reducing the complexity of the data to better depict the sources of variation. This revealed proteomic measures and CSF core $\mathrm{AD}$ biomarkers as the main contributors to the variance with both having very similar contributions to variance, albeit from 768 proteins versus 3 AD biomarkers. The biomarker contribution was expected as our sample contains a large proportion of participants with $\mathrm{AD}$, each displaying CSF AD biomarkers significantly different from subjects without AD. The large contribution of proteomics to variance could derive, at least in part, from the fact that protein expression levels reflect the effects of different environments, life style, health conditions, and genetic backgrounds; all factors potentially affecting protein expression and regulation [73]. Nonetheless, MOFA analysis identified 21 proteins with previously reported association to $\mathrm{AD}$, suggesting, along with the associations with $\mathrm{AD}$ biomarkers observed here, that protein contribution to variance is linked to $\mathrm{AD}$ pathology. These findings further show that the MOFA approach can accurately disentangle the inter-individual heterogeneity driven by $\mathrm{AD}$ pathology and differentiate between individual (i.e., not repeated in 
Table 6 Analytes associated with latent factors

\begin{tabular}{|c|c|c|c|c|}
\hline LF & Analyte & Full name & Entry\# & Previously reported $A D$ association \\
\hline \multicolumn{5}{|c|}{ Proteomics } \\
\hline 1 & NRN1 & Neuritin isoform 1 precursor & Q9NPD7 & \\
\hline 1 & SMS & Spermine synthase & P52788 & Yes [37] \\
\hline 1 & $\mathrm{NXPH} 4$ & Neurexophilin-4 & O95158 & \\
\hline 1 & LTBP1 & Latent-transforming growth factor beta-binding protein 1 & Q14766 & \\
\hline 1 & CLUS & Clusterin & P10909 & Yes [38] \\
\hline 1 & NPDC1 & Neural proliferation differentiation and control protein 1 & Q9NQX5 & \\
\hline 1 & PNOC & Prepronociceptin & Q13519 & \\
\hline 1 & DYL2 & Dynein light chain 2, cytoplasmic & Q96FJ2 & \\
\hline 1 & PDGFB & Platelet-derived growth factor subunit B & P01127 & Yes [39] \\
\hline 1 & SAP3 & Sphingolipid activator protein 3 & P17900 & \\
\hline 1 & MT1E & Metallothionein-1E & P04732 & Yes [40] \\
\hline 1 & PCSK1 & Neuroendocrine convertase 1 & P29120 & Yes [41] \\
\hline 1 & TAGL & Transgelin-2 & P37802 & Yes [42] \\
\hline 1 & MT3 & Metallothionein-3 & P25713 & Yes [40] \\
\hline 1 & LY6H & Lymphocyte antigen $6 \mathrm{H}$ & O94772 & \\
\hline 2 & SAMP & Spindle-associated membrane protein 1 & Q5SNT2 & \\
\hline 2 & VTNC & Vitronectin & P04004 & Yes [43] \\
\hline 2 & KNG1 & Kininogen-1 & P01042 & \\
\hline 2 & FETUA & Alpha-2-HS-glycoprotein & P02765 & Yes [44] \\
\hline 2 & HELZ & Probable helicase with zinc finger domain & P42694 & \\
\hline 2 & PLMN & Plasminogen & P00747 & Yes [45] \\
\hline 2 & PGRP2 & $\mathrm{N}$-acetylmuramoyl-L-alanine amidase & Q96PD5 & \\
\hline 2 & AFAM & Afamin & P43652 & Yes [44] \\
\hline 2 & $\mathrm{ITIH} 1$ & Inter-alpha-trypsin inhibitor heavy chain $\mathrm{H} 1$ & P19827 & Yes [46] \\
\hline 2 & $\mathrm{CO} 8 \mathrm{~B}$ & Complement component C8 beta chain & P07358 & Yes [47] \\
\hline 2 & FIBA & Fibrinogen alpha chain & P02671 & Yes [48] \\
\hline 2 & $\mathrm{CO} 6$ & Complement component C6 & P13671 & Yes [47] \\
\hline 2 & $\mathrm{ITH} 4$ & Inter-alpha-trypsin inhibitor heavy chain $\mathrm{H} 4$ & Q14624 & Yes [46] \\
\hline 3 & EPDR1 & Mammalian ependymin-related protein 1 & Q9UM22 & \\
\hline 3 & SIAE & Sialate O-acetylesterase & Q9HAT2 & \\
\hline 4 & X1433Z & 14-3-3 protein zeta/delta & P63104 & Yes [49] \\
\hline 4 & S10A6 & Protein S100-A6 & P06703 & Yes [50] \\
\hline 4 & PRDX6 & Peroxiredoxin-6 & P30041 & Yes [51] \\
\hline 5 & VTM2A & $\mathrm{V}$-set and transmembrane domain-containing protein $2 \mathrm{~A}$ & Q8TAG5 & \\
\hline 5 & S10A6 & Protein S100-A6 & P06703 & Yes [50] \\
\hline 5 & CMGA & Chromogranin-A & P10645 & Yes [52] \\
\hline 5 & ZP2 & Zona pellucida sperm-binding protein 2 & Q05996 & \\
\hline 5 & SLIK1 & SLIT and NTRK-like protein 1 & Q96PX8 & Yes [53] \\
\hline \multicolumn{5}{|c|}{ Neuroinflammation } \\
\hline 1 & sVCAM-1 & Circulating vascular cell adhesion molecule-1 & P19320 & Yes [54] \\
\hline 1 & IL-15 & Interleukin-15 & P40933 & Yes [54] \\
\hline 1 & sICAM-1 & Soluble intracellular adhesion molecule-1 & P05362 & Yes [54] \\
\hline 2 & SAA & Serum amyloid $A$ & P0DJI8 & Yes [55] \\
\hline
\end{tabular}


Table 6 Analytes associated with latent factors (Continued)

\begin{tabular}{|c|c|c|c|c|}
\hline LF & Analyte & Full name & Entry\# & Previously reported $A D$ association \\
\hline 2 & PIGF_1R & Insulin-like growth factor 1 receptor & P08069 & Yes [56] \\
\hline 3 & PIGF_1R & Insulin-like growth factor 1 receptor & P08069 & Yes [56] \\
\hline 4 & $\mathrm{IL}-16$ & Interleukin-16 & Q14005 & Yes [57] \\
\hline 5 & MCP-1 & Monocyte chemoattractant protein-1 & P13500 & Yes [54] \\
\hline 5 & PIGF_1R & Insulin-like growth factor 1 receptor & P08069 & Yes [56] \\
\hline \multicolumn{5}{|c|}{ One-carbon metabolism } \\
\hline 1 & MTHF & 5-methyltetrahydrofolate & 20,612 & Yes [58] \\
\hline 1 & $\mathrm{SAH}$ & S-adenosyl-L-homocysteine & 16,680 & Yes [59] \\
\hline 2 & CYST & Total cysteine & 15,356 & Yes [60] \\
\hline 3 & $\mathrm{SAH}$ & S-adenosyl-L-homocysteine & 16,680 & Yes [59] \\
\hline 4 & CYST & Total cysteine & 15,356 & Yes [60] \\
\hline 5 & CYST & Total cysteine & 15,356 & Yes [60] \\
\hline \multicolumn{5}{|c|}{ Metabolomics } \\
\hline 1 & $\mathrm{~N} / \mathrm{A}$ & Glycoproteins & 17,089 & Yes [61] \\
\hline 2 & $\mathrm{~N} / \mathrm{A}$ & Alanine & 16,449 & Yes [62] \\
\hline 2 & N/A & Valine & 27,266 & \\
\hline 2 & N/A & Glycoproteins & 17,089 & Yes [61] \\
\hline 3 & N/A & Inositol & 24,848 & \\
\hline 4 & N/A & Glycoproteins & 17,089 & Yes [61] \\
\hline 5 & N/A & Formic acid & 30,751 & \\
\hline 5 & 569 & Unidentified metabolite & N/A & N/A \\
\hline 5 & N/A & Acetoacetic acid & 15,344 & Yes [63] \\
\hline \multicolumn{5}{|c|}{ Lipidomics } \\
\hline 1 & PC 32:0 & 1,2-Dihexadecanoyl-sn-glycero-3-phosphocholine & N/A & Yes [64] \\
\hline 2 & SE 27:1 18:2 & Cholesteryl ester & N/A & \\
\hline 3 & PC 32:0 & 1,2-Dihexadecanoyl-sn-glycero-3-phosphocholine & N/A & Yes [64] \\
\hline 4 & SE 27:1 18:2 & Cholesteryl ester & N/A & \\
\hline 4 & SE 27:1 20:4 & Cholesteryl ester & $\mathrm{N} / \mathrm{A}$ & \\
\hline 4 & SE 27:1 16:0 & Cholesteryl ester & $\mathrm{N} / \mathrm{A}$ & \\
\hline 5 & LPG 20:1 & 1-(11Z-eicosenoyl)-glycero-3-phospho-(1'-sn-glycerol) & N/A & \\
\hline
\end{tabular}

CSF biomolecules significantly associated with the LFs within the MOFA model and whether they have been previously associated with AD. Entry\# denotes the analyte identifier within the UniProt database (for proteomics and neuroinflammation) or ChEBI database (for other analytes)

the dataset) and cohort heterogeneity (i.e., underlying changes in many participants). Conversely, the metabolomic dataset was only responsible for a small amount of the cohort heterogeneity $(3.7 \%)$, a possible explanation being that it represents individual heterogeneity for the most part caused by the environment, disease processes, or nutritional habits. This low contribution of metabolomics to variance could also result from the lower dimensionality of the metabolomics dataset as molecules within had lower concentrations compared to molecules in the other modalities. Yet, despite this low level of variance, our model was able to correctly retrieve metabolites previously reported in association with $\mathrm{AD}$, underlining the sensitivity of the model. This is further supported by the ability of our approach to determine a four-molecule signature that improves the prediction of AD pathology.

\section{Associations between $L F$ and specific aspects of $A D$ pathology}

Our analysis revealed that LFs $1-3$ were primarily associated with neuronal injury while LF4 and LF5 were mainly associated with amyloid pathology. In addition, both Tau and P-Tau were negatively associated with LF3, while $A \beta_{1-42}$ presented a positive, albeit weaker, association with this same LF. Conversely, Tau and P-Tau were positively while $A \beta_{1-42}$ was strongly negatively associated with LF4. Whether the LF3 and LF4, that show 

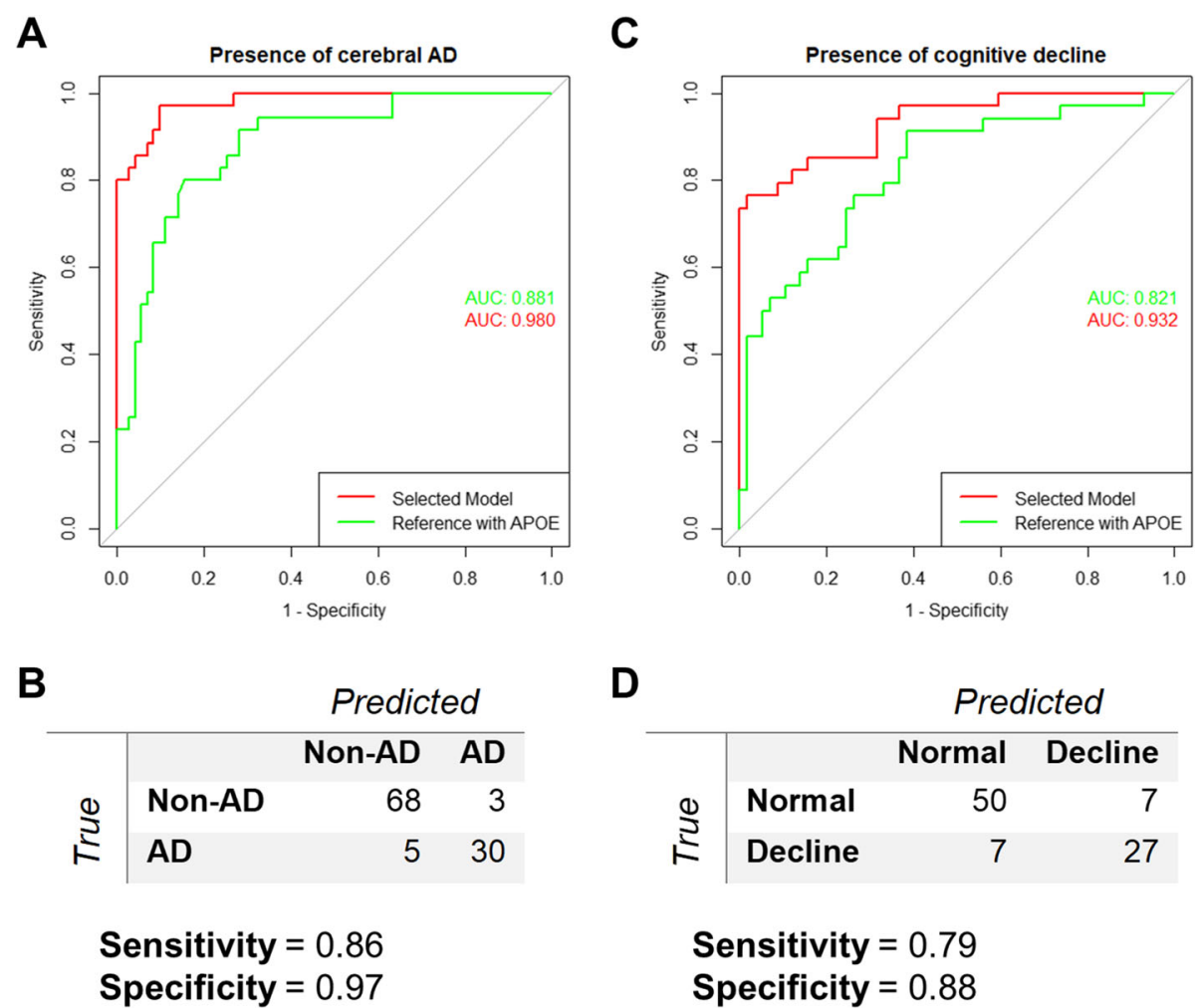

$$
\begin{aligned}
& \text { Sensitivity }=0.86 \\
& \text { Specificity }=0.97
\end{aligned}
$$$$
\text { Specificity }=0.88
$$

Fig. 5 Clinical predictions. Binary logistic regression models to improve clinical predictions. a ROC curves and AUCs for the reference model including APOE status (green) and the final prediction model of AD pathology (red) obtained after addition of four analytes (14-3-3 zeta/delta, clusterin, interleukin-15, and transgelin-2) selected by the MOFA model. b Confusion matrix of the final prediction model of AD. $\mathbf{c}$ ROC curves and AUCs for the reference model including APOE status (green) and the final prediction model of cognitive decline (red) obtained after addition of four analytes (14-3-3 zeta/delta, clusterin, cholesteryl ester 27:1 16:0 and monocyte chemoattractant protein-1) selected by the MOFA model. $\mathbf{d}$ Confusion matrix of the final prediction model of cognitive decline

congruent relationships with amyloid aggregation, tau pathology and neurodegeneration, may be particularly relevant for $\mathrm{AD}$ remains to be confirmed. The finding that the zeta/delta isoform of protein 14-3-3, selected in LF4, is associated with all AD CSF biomarkers along with its contribution to predictive models of cerebral $\mathrm{AD}$ is in line with the hypothesis that these LFs are involved in AD pathology.

\section{Interactions between LFs and 'omics modalities}

Besides the identification of molecular profiles and metabolic pathways alterations associated with $\mathrm{AD}$, our approach offers the unique ability to disentangle how components of individual LFs interact with each other to explain variance within the cohort. This not only reveals specific interactions between subsets of molecules and particular metabolic pathways but also offers a unique view into how multiple biological levels interact in the context of AD pathology and how they are related to specific aspects of the pathology. In addition, this approach has the advantage of not being biased towards any known biological alteration of $\mathrm{AD}$ pathology or giving any particular weight to a specific molecule or metabolic pathway. In the context of $\mathrm{AD}$, this could lead to the identification of pathways and alterations not directly related to the core features of AD pathology, better reflecting the heterogeneity of the disease. The presence of clusters within each LF also suggests specific groups of molecules interacting with each other across LFs. A more comprehensive analysis of the role of these clusters of analytes may be addressed in future studies.

\section{Novel associations uncovered by the MOFA model}

The MOFA model uncovered additional relationships not revealed by other analysis exploration paradigms since it does not only consider molecules from one modality but the whole dataset from different 'omics. These additional findings may result from the downstream effects of these molecules or from interactions with other modalities. These include the association of total cysteine with CSF $A \beta_{1-42}$, Tau, and P-Tau or the association of kininogen-1 (KNG1) with Tau and P-Tau. While cysteine was previously linked with $A D$ [60], KNG-1 has been associated with other 


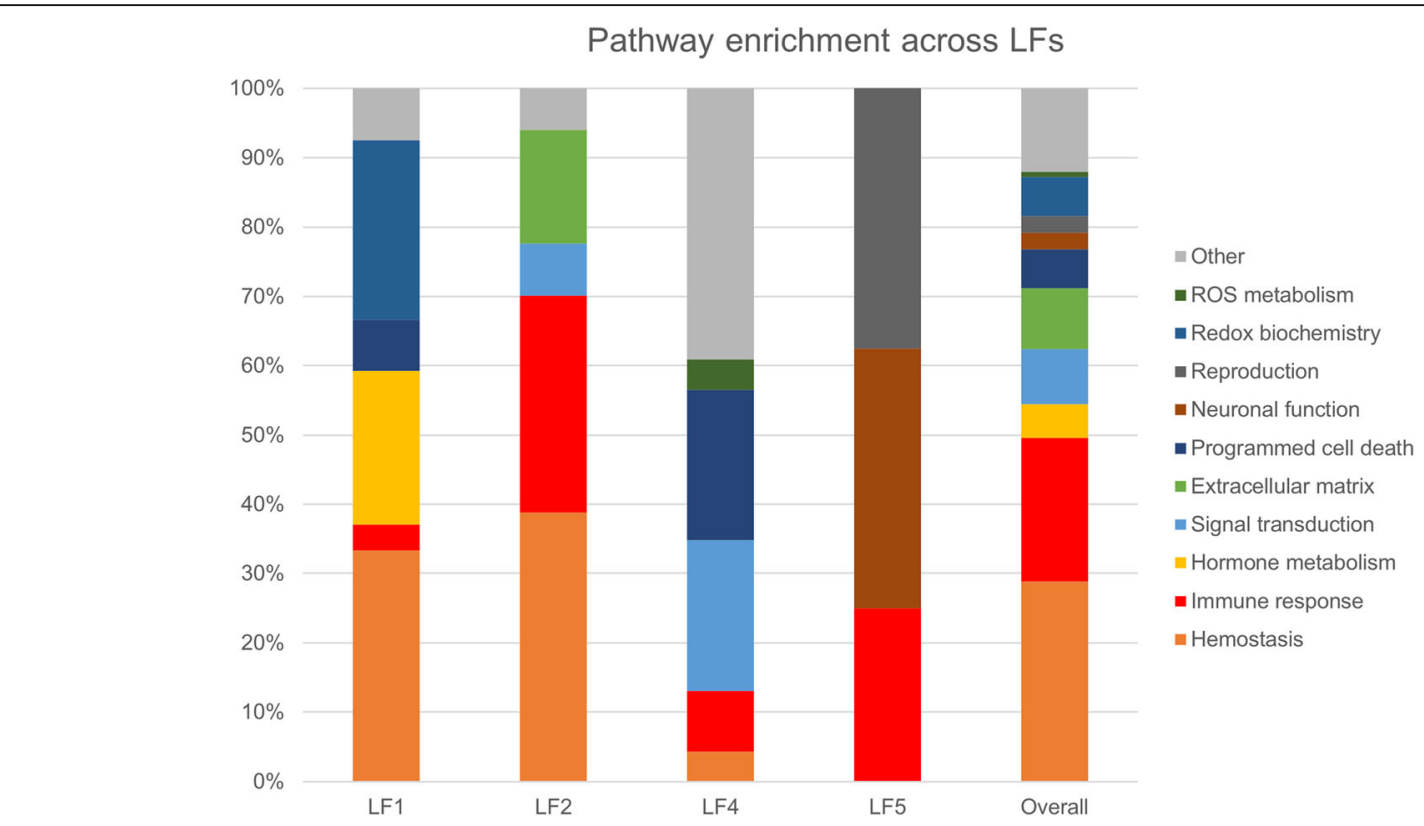

Fig. 6 Pathway enrichment. Pathway enrichment analysis of identified proteins across LFs and overall. The number of over-represented categories within each LF (expressed as a percentage) as well as across all LFs is represented. NB: the low number of analytes associated with LF3 did not allow for an enrichment analysis

neurodegenerative disorders [74], but its association with $\mathrm{AD}$, and in particular tau pathology and neuronal injury, is novel. The MOFA model also identified molecules previously associated with cognitive impairment, such as dynein light-chain 2, cytoplasmic (DYL2), and neurexophilin-4 (NXPH4). Both were associated with LF1 and with cognitive impairment. DYL2 is thought to regulate dynein function [75] and maintain cytoskeletal structure, therefore regulating synaptic function [76]. NXPH4 structurally resembles neurexophilin-1, an $\alpha$-neurexin ligand, which promotes adhesion between dendrites and axons and modulates specific cerebellar synapses and motor functions [77]. Altered levels of these proteins may therefore be associated with neurodegeneration processes and related to cognitive impairment in AD. Another novel analyte we identified is the cholesteryl ester SE 27:1 16:0. While links between phosphatidylcholine metabolism and $\mathrm{AD}$ in general [78] and PC 32:0 in particular [64] have been previously reported, to our knowledge, cholesteryl esters have not previously been associated with AD pathology. In our MOFA model, this cholesteryl ester was strongly correlated to LF4, suggesting a role in amyloid pathology. These molecules were also associated with cognitive performance as measured by MMSE. Together, these results demonstrate the capacity of integrative multi-omics to provide additional insights into the relationship of molecular alterations with specific aspects of the AD pathology.

\section{Prediction of AD pathology and cognitive decline using MOFA-selected molecules}

Molecular signatures associated with $\mathrm{AD}$ or predictive of cognitive decline were derived from our model. Both signatures contain four molecules each, taken from multiple biological levels and significantly improved prediction performance when added to reference models. The four molecules from the combination related to $\mathrm{AD}$ pathology have each been associated with AD previously [38, 42, 49, 54]. From the molecule combination that improved prediction of cognitive decline, three molecules have been linked to cognitive decline in previous reports [79-81], while one molecule, cholesteryl ester 27:1 16:0 was not. Both signatures also share two common molecules, protein 14-3-3 zeta/delta and clusterin, suggesting these belong to common biological pathways both associated with $\mathrm{AD}$ and relevant for cognitive decline. Cholesteryl ester 27:1 16:0 and monocyte chemoattractant protein-1 may indicate pathway alterations without a strong and direct link to core AD pathology but having impact on the rapidity of cognitive decline. These predictive models also demonstrate the ability of this approach to identify biomarker candidates for both $\mathrm{AD}$ pathology and cognitive decline. Additional investigation and validation in independent cohorts is required before possible clinical use.

\section{Infer pathway relationships with AD pathology}

One important strength of the MOFA approach is that it enables addressing the relationship between multiple 
biological pathways and associate them with sources of variance (i.e., LFs). Using over-representation of metabolic pathways, we were able to show that individual $\mathrm{LFs}$, and the main related pathological aspects of $\mathrm{AD}$ (i.e., amyloid aggregation, neurodegeneration and tau pathology) are associated with distinct pathways. Hemostasis and immune response were the most overrepresented. Only the immune response was associated with all LFs in which individual pathways could be identified. LF1 and LF2 presented a significant enrichment in biomolecules implicated in hemostasis, suggesting an association between this pathway and neuronal injury, and tau pathology. While an association between hemostasis and amyloid pathology pathway was previously described [82], in particular related to expression of amyloid precursor protein and release of $A \beta$ [83], there have also been recent reports of an association between Tau and hemostasis [84]. Molecules involved in the extracellular matrix were significantly enriched in LF2, also suggesting an association with tau-related pathology, in line with previous reports [85]. However, this pathway was not detected within LF1 or other LFs. We therefore hypothesize that the molecules involved are those presenting a specific pattern of association with LF2, such as PC 32:0, PC 34:1, LPA 18:3, and TAG 54:3. Neuronal function was confined to associations with LF5, suggesting little variation and differences in signal transmission and synaptic function across the cohort since this LF only explained $8 \%$ of the variance. Nonetheless, this result suggests an association with amyloid pathology, which is in accordance with previous findings of amyloid being released in an activity-dependent fashion from neurons and modulating synaptic function and plasticity $[86,87]$. Overall, the enriched metabolic pathways suggest that AD pathology affects not only pathways related to neuronal biological systems but is linked to a broader spectrum of metabolic dysfunctions.

\section{Limitations}

A limitation of this study is the different amounts of analytes measured by individual quantification method (i.e., $>500$ proteins measured vs. $<100$ metabolites/lipids for example) resulting from methodological differences. This approach prevents measuring the relative importance of the analytes or their combinations but allows the identification of altered pathways or molecular signatures from different modalities. Furthermore, since the data entered in the MOFA analyses did not include information regarding clinical stages, stage-specific alterations have not been addressed. Also, the inclusion of some targeted analysis results in the multi-omics models may be considered as a limitation. While the proteomic and lipidomic dataset are hypothesis-free measurements and the study could be limited to this data, we chose to include further available modalities. In particular, we considered neuroinflammation and one-carbon metabolism given their previously reported associations with $\mathrm{AD}$ and relevance for brain metabolism. However, the replication of these and other previously reported associations in our MOFA model along with the ability of the MOFA selected marker combinations to improve prediction of $\mathrm{AD}$ and of cognitive decline support the validity of the new findings revealed in the present study.

\section{Conclusions}

Here, applying integrative multi-omics in $\mathrm{AD}$, we have identified five axes of variation within a cohort of individuals with normal cognition or with cognitive impairment. These five LFs were associated with different aspects of the core AD pathology. We confirmed several previously reported associations with $\mathrm{AD}$ and identified new molecular patterns interrelated within each LF. Additionally, we identified molecular biomarker signatures improving the diagnosis of $\mathrm{AD}$ pathology and the prediction of future cognitive decline. Furthermore, using pathway enrichment analysis, we have revealed metabolic pathways represented within single LFs and explored specific relationships with markers of amyloid pathology, neuronal injury, and tau hyperphosphorylation. These findings demonstrate the added value of integrative multi-omics analysis to uncover interrelated pathway alterations in $\mathrm{AD}$ and its ability to identify biomarker combinations that may be used in clinical practice. This is relevant for the development of both personalized diagnosis and tailored therapeutic interventions in $\mathrm{AD}$.

\section{Abbreviations}

$A \beta_{1-42}$ : CSF beta-amyloid 1-42; AD: Alzheimer's disease; AIC: Akaike information criterion; AUC: Area under the curve; CDR: Clinical Dementia Rating; CDR-SoB: CDR sum of boxes; CSF: Cerebrospinal fluid; ${ }^{1} \mathrm{H}$ NMR: Proton nuclear magnetic resonance; LF: Latent factor; $\mathrm{MCl}$ : Mild cognitive impairment; MMSE: Mini mental state examination; MOFA: Multi-omics factor analysis; MS: Mass spectrometry; P-Tau: Tau phosphorylated at threonine 181; Tau: Total-tau

\section{Supplementary Information}

The online version contains supplementary material available at https://doi. org/10.1186/s13195-021-00814-7.

Additional file 1. Supplementary methods used in this study, including additional details on omics techniques used for quantification, validation of the MOFA model and association with clinical measurements.

Additional file 2: Additional Figures, including a correlation matrix analysis of latent factors (Figure S1) and prediction of CSF AD biomarkers using the trained MOFA model (Figure S2).

Additional file 3: Additional Tables, including coarse-grain categories used for pathway enrichment (Table S1), relationship between selected CSF molecules and latent factors (Table S2), correlations between selected proteins and lipids with CDR-SoB and MMSE scores (Tables S3) and CSF proteins presenting an association with cognitive impairment (Table S4). 


\section{Acknowledgements}

We thank Antonio Nuñez Galindo, John Corthésy, Ornella Cominetti, Delphine Morin-Rivron, Seu-Ping Guiraud, Laeticia Da Siva, François-Pierre Martin, Sofia Moço, Sebastiano Collino, Martin Kussmann, Sylviane Métairon, Julien Marquis, Patrick Descombes, and Richard Kirkland for biological and genetic analyses.

\section{Authors' contributions}

CC and JP designed the study. LD, MM, and GB designed the experiments and analyzed multi-omics data. CC performed the data processing and bioinformatics analysis and generated text and figures for the manuscript. CC, $L D, M M, G B$, and JP designed and established the study cohorts and contributed to writing the paper. JP supervised and guided the project. CC and JP wrote the manuscript. All authors revised and approved the final draft.

\section{Funding}

This work was supported by grants from the Swiss National Research Foundation (to JP, SNF 320030_141179), Synapsis Foundation - Alzheimer Research Switzerland (to JP, grant number 2017-PI01) and funding from the Nestlé Institute of Health Sciences.

\section{Availability of data and materials}

Proteomics data used for this study was previously deposited to the ProteomeXchange Consortium (http://proteomecentral.proteomexchange. org) via the PRIDE partner repository and is available with the identifier PXD009589. The other datasets used and/or analyzed during the current study are available from the corresponding author on reasonable request.

\section{Declarations}

\section{Ethics approval and consent to participate}

The local ethics committee of Vaud, Switzerland, approved this study (No. 171/2013), and all participants or their legal representatives provided written informed consent.

\section{Consent for publication}

Not applicable

\section{Competing interests}

JP received consultation honoraria from Nestle Institute of Health Sciences, Innovation Campus, EPFL, Lausanne, Switzerland, Ono Pharma, and from Fujirebio Europe. The other authors declare no potential conflicts of interest.

\section{Author details}

'Institute for Regenerative Medicine, University of Zürich, Wagistrasse 12, 8952 Schlieren, Switzerland. ${ }^{2}$ Nestlé Institute of Health Sciences, Nestlé Research, EPFL Innovation Park, 1015 Lausanne, Switzerland. ${ }^{3}$ Nestlé Institute of Food Safety \& Analytical Sciences, Nestlé Research, EPFL Innovation Park, 1015 Lausanne, Switzerland. ${ }^{4}$ Institut des Sciences et Ingénierie Chimiques, Ecole Polytechnique Fédérale de Lausanne, 1015 Lausanne, Switzerland. 5 Institute of Clinical Chemistry, University Hospital Bern, Bern, Switzerland. ${ }^{6}$ Department of Neurology, NIA-Layton Aging and Alzheimer's Disease Center, Oregon Health \& Science University, Portland, USA. 'Old Age Psychiatry, Centre Hospitalier Universitaire Vaudois, Rue du Bugnon 46, 1011 Lausanne, Switzerland. ${ }^{8}$ Department of Geriatric Psychiatry, University Hospital of Psychiatry Zürich, Centre for Gerontopsychiatric Medicine, Minervastrasse 145, P.O. Box 341, 8032 Zürich, Switzerland.

\section{Received: 30 November 2020 Accepted: 23 March 2021}

\section{Published online: 01 April 2021}

\section{References}

1. Leng F, Edison P. Neuroinflammation and microglial activation in Alzheimer disease: where do we go from here? Nat Rev Neurol. 2021;17(3):157-72.

2. Wong MW, Braidy N, Poljak A, Pickford R, Thambisetty M, Sachdev PS. Dysregulation of lipids in Alzheimer's disease and their role as potential biomarkers. Alzheimers Dement. 2017;13(7):810-27. https://doi.org/10.1016/j. jalz.2017.01.008.

3. Dayon L, Guiraud SP, Corthesy J, Da Silva L, Migliavacca E, Tautvydaite D, et al. One-carbon metabolism, cognitive impairment and CSF measures of
Alzheimer pathology: homocysteine and beyond. Alzheimers Res Ther. 2017;9(1):43. https://doi.org/10.1186/s13195-017-0270-x.

4. de Leeuw FA, Peeters CFW, Kester MI, Harms AC, Struys EA, Hankemeier T, et al. Blood-based metabolic signatures in Alzheimer's disease. Alzheimers Dement (Amst). 2017;8(1):196-207. https://doi.org/10.1016/j.dadm.2017.07.006.

5. Yan X, Hu Y, Wang B, Wang S, Zhang X. Metabolic dysregulation contributes to the progression of Alzheimer's disease. Front Neurosci. 2020;14:530219. https://doi.org/10.3389/fnins.2020.530219.

6. Sancesario GM, Bernardini S. Alzheimer's disease in the omics era. Clin Biochem. 2018;59:9-16. https://doi.org/10.1016/j.clinbiochem.2018.06.011.

7. Dayon L, Cominetti O, Wojcik J, Galindo AN, Oikonomidi A, Henry H, Migliavacca E, Kussmann M, Bowman GL, Popp J. Proteomes of paired human cerebrospinal fluid and plasma: relation to blood-brain barrier permeability in older adults. J Proteome Res. 2019;18(3):1162-74. https://doi. org/10.1021/acs.jproteome.8b00809.

8. van der Velpen V, Teav T, Gallart-Ayala H, Mehl F, Konz I, Clark C, Oikonomidi A, Peyratout G, Henry H, Delorenzi M, Ivanisevic J, Popp 」. Systemic and central nervous system metabolic alterations in Alzheimer's disease. Alzheimers Res Ther. 2019;11(1):93. https://doi.org/10.1186/s13195019-0551-7.

9. Huang $S$, Chaudhary K, Garmire LX. More is better: recent progress in multiomics data integration methods. Front Genet. 2017;8:84. https://doi.org/1 0.3389/fgene.2017.00084

10. Su M-W, Chang C-K, Lin C-W, Ling S-J, Hsiung C-N, Chu H-W, Wu PE, Shen $\mathrm{CY}$. Blood multiomics reveal insights into population clusters with low prevalence of diabetes, dyslipidemia and hypertension. PLoS One. 2020; 15(3):e0229922. https://doi.org/10.1371/journal.pone.0229922.

11. Chalikiopoulou C, Jenko Bizjan B, Leventopoulos G, Smaili K, Blagus T, Menti A, et al. Multiomics analysis coupled with text mining identify novel biomarker candidates for recurrent cardiovascular events. OMICS. 2020;24(4): 205-15.

12. Wang B, Lunetta KL, Dupuis J, Lubitz SA, Trinquart L, Yao L, Ellinor PT, Benjamin EJ, Lin H. Integrative omics approach to identifying genes associated with atrial fibrillation. Circ Res. 2020;126(3):350-60. https://doi. org/10.1161/CIRCRESAHA.119.315179.

13. Badhwar A, McFall GP, Sapkota S, Black SE, Chertkow H, Duchesne S, et al. A multiomics approach to heterogeneity in Alzheimer's disease: focused review and roadmap. Brain. 2020;143(5):1315-31.

14. Argelaguet R, Velten B, Arnol D, Dietrich S, Zenz T, Marioni JC, Buettner F, Huber W, Stegle O. Multi-omics factor analysis-a framework for unsupervised integration of multi-omics data sets. Mol Syst Biol. 2018;14(6): e8124. https://doi.org/10.15252/msb.20178124.

15. Mirza B, Wang W, Wang J, Choi H, Chung NC, Ping P. Machine learning and integrative analysis of biomedical big data. Genes 2019;10(2):87.

16. Argelaguet R, Arnol D, Bredikhin D, Deloro Y, Velten B, Marioni JC, Stegle O. MOFAt: a statistical framework for comprehensive integration of multimodal single-cell data. Genome Biol. 2020;21(1):111. https://doi.org/10.1186/ s13059-020-02015-1.

17. Folstein MF, Folstein SE, McHugh PR. Mini-mental state. J Psychiatr Res. 1975; 12(3):189-98 Available from: URL: https://pubmed.ncbi.nIm.nih.gov/1202204/.

18. Morris JC. The Clinical Dementia Rating (CDR): current version and scoring rules. Neurology. 1993:43(11):2412-4. https://doi.org/10.1212/ WNL.43.11.2412-a.

19. Tautvydaite D, Antonietti JP, Henry H, von Gunten A, Popp J. Relations between personality changes and cerebrospinal fluid biomarkers of Alzheimer's disease pathology. J Psychiatr Res. 2017;90:12-20. https://doi. org/10.1016/j.jpsychires.2016.12.024.

20. Dayon L, Núñez Galindo A, Cominetti O, Corthésy J, Kussmann M. A highly automated shotgun proteomic workflow: clinical scale and robustness for biomarker discovery in blood. Methods Mol Biol. 2017;1619:433-49.

21. Dayon L, Núñez Galindo A, Corthésy J, Cominetti O, Kussmann M. Comprehensive and scalable highly automated MS-based proteomic workflow for clinical biomarker discovery in human plasma. J Proteome Res. 2014;13(8):3837-45. https://doi.org/10.1021/pr500635f.

22. Dayon L, Sanchez J-C. Relative protein quantification by MS/MS using the tandem mass tag technology. Methods Mol Biol. 2012;893:115-27. https:// doi.org/10.1007/978-1-61779-885-6_9

23. Dayon L, Wojcik J, Nunez Galindo A, Corthesy J, Cominetti O, Oikonomidi A et al. Plasma proteomic profiles of cerebrospinal fluid-defined Alzheimer's disease pathology in older adults. J Alzheimers Dis. 2017;60(4):1641-52. https://doi.org/10.3233/JAD-170426. 
24. Dayon L, Nunez Galindo A, Wojcik J, Cominetti O, Corthesy J, Oikonomidi A, et al. Alzheimer disease pathology and the cerebrospinal fluid proteome. Alzheimers Res Ther. 2018;10(1):66. https://doi.org/10.1186/s13195-018-03 97-4.

25. Bettcher BM, Johnson SC, Fitch R, Casaletto KB, Heffernan KS, Asthana S, Zetterberg H, Blennow K, Carlsson CM, Neuhaus J, Bendlin BB, Kramer JH. Cerebrospinal fluid and plasma levels of inflammation differentially relate to CNS markers of Alzheimer's disease pathology and neuronal damage. J Alzheimers Dis. 2018;62(1):385-97. https://doi.org/10.3233/JAD-170602.

26. Surma MA, Herzog R, Vasilj A, Klose C, Christinat N, Morin-Rivron D, Simons K, Masoodi M, Sampaio JL. An automated shotgun lipidomics platform for high throughput, comprehensive, and quantitative analysis of blood plasma intact lipids. Eur J Lipid Sci Technol. 2015;117(10):1540-9. https://doi.org/1 0.1002/ejtt.201500145.

27. Lauria M, Persico M, Dordevic N, Cominetti O, Matone A, Hosking J, Jeffery A, Pinkney J, da Silva L, Priami C, Montoliu I, Martin FP. Consensus clustering of temporal profiles for the identification of metabolic markers of prediabetes in childhood (EarlyBird 73). Sci Rep. 2018;8(1):1393. https://doi. org/10.1038/s41598-017-19059-2

28. Guiraud SP, Montoliu I, Da Silva L, Dayon L, Galindo AN, Corthesy J, et al. High-throughput and simultaneous quantitative analysis of homocysteinemethionine cycle metabolites and co-factors in blood plasma and cerebrospinal fluid by isotope dilution LC-MS/MS. Anal Bioanal Chem. 2017; 409(1):295-305. https://doi.org/10.1007/s00216-016-0003-1.

29. Bowman GL, Dayon L, Kirkland R, Wojcik J, Peyratout G, Severin IC, Henry H, Oikonomidi A, Migliavacca E, Bacher M, Popp J. Blood-brain barrier breakdown, neuroinflammation, and cognitive decline in older adults. Alzheimers Dement. 2018;14(12):1640-50. https://doi.org/10.1016/j.jalz.2018. 06.2857.

30. Popp J, Oikonomidi A, Tautvydaite D, Dayon L, Bacher M, Migliavacca E, et al. Markers of neuroinflammation associated with Alzheimer's disease pathology in older adults. Brain Behav Immun. 2017;62:203-11. https://doi. org/10.1016/j.bbi.2017.01.020.

31. Oikonomidi A, Tautvydaite D, Gholamrezaee MM, Henry H, Bacher M, Popp J. Macrophage migration inhibitory factor is associated with biomarkers of Alzheimer's disease pathology and predicts cognitive decline in mild cognitive impairment and mild dementia. J Alzheimers Dis. 2017;60(1):27381. https://doi.org/10.3233/JAD-170335.

32. Oikonomidi A, Lewczuk P, Kornhuber J, Smulders $Y$, Linnebank M, Semmler A, Popp J. Homocysteine metabolism is associated with cerebrospinal fluid levels of soluble amyloid precursor protein and amyloid beta. J Neurochem. 2016;139(2):324-32. https://doi.org/10.1111/jnc.13766.

33. Chakrabarti A, Eiden M, Morin-Rivron D, Christinat N, Monteiro JP, Kaput J, et al. Impact of multi-micronutrient supplementation on lipidemia of children and adolescents. Clin Nutr. 2020;39(7):2211-9.

34. Friedman J, Hastie T, Tibshirani R. Regularization paths for generalized linear models via coordinate descent. J Stat Softw. 2010;33(1):1-22.

35. Uniprot Consortium. UniProt: a worldwide hub of protein knowledge. Nucleic Acids Res. 2019;47(D1):D506-15.

36. Jassal B, Matthews L, Viteri G, Gong C, Lorente P, Fabregat A, Sidiropoulos K, Cook J, Gillespie M, Haw R, Loney F, May B, Milacic M, Rothfels K, Sevilla C, Shamovsky V, Shorser S, Varusai T, Weiser J, Wu G, Stein L, Hermjakob H, D'Eustachio P. The reactome pathway knowledgebase. Nucleic Acids Res. 2019. https://doi.org/10.1093/nar/gkz1031.

37. Liu P, Fleete MS, Jing Y, Collie ND, Curtis MA, Waldvogel HJ, Faull RLM, Abraham WC, Zhang H. Altered arginine metabolism in Alzheimer's disease brains. Neurobiol Aging. 2014;35(9):1992-2003. https://doi.org/10.1016/j. neurobiolaging.2014.03.013.

38. Miners JS, Clarke P, Love S. Clusterin levels are increased in Alzheimer's disease and influence the regional distribution of Abeta. Brain Pathol (Zurich, Switzerland). 2017;27(3):305-13.

39. Bjorkqvist M, Ohlsson M, Minthon L, Hansson O. Evaluation of a previously suggested plasma biomarker panel to identify Alzheimer's disease. PLoS One. 2012;7(1):e29868. https://doi.org/10.1371/journal.pone.0029868.

40. Juarez-Rebollar D, Rios C, Nava-Ruiz C, Mendez-Armenta M. Metallothionein in brain disorders. Oxidative Med Cell Longev. 2017;2017:5828056.

41. Hokama M, Oka S, Leon J, Ninomiya T, Honda H, Sasaki K, et al. Altered expression of diabetes-related genes in Alzheimer's disease brains: the Hisayama study. Cereb Cortex (New York, N.Y. : 1991). 2014;24(9):2476-88.

42. Muller T, Concannon CG, Ward MW, Walsh CM, Tirniceriu AL, Tribl F, et al. Modulation of gene expression and cytoskeletal dynamics by the amyloid precursor protein intracellular domain (AICD). Mol Biol Cell. 2007;18(1):20110. https://doi.org/10.1091/mbc.e06-04-0283.

43. Kiddle SJ, Thambisetty M, Simmons A, Riddoch-Contreras J, Hye A, Westman E, Pike I, Ward M, Johnston C, Lupton MK, Lunnon K, Soininen H, Kloszewska I, Tsolaki M, Vellas B, Mecocci P, Lovestone S, Newhouse S, Dobson R, for the Alzheimers Disease Neuroimaging Initiative. Plasma based markers of [11C] PiB-PET brain amyloid burden. PLoS One. 2012;7(9):e44260. https://doi. org/10.1371/journal.pone.0044260.

44. Kitamura Y, Usami R, Ichihara S, Kida H, Satoh M, Tomimoto H, Murata M, Oikawa S. Plasma protein profiling for potential biomarkers in the early diagnosis of Alzheimer's disease. Neurol Res. 2017;39(3):231-8. https://doi. org/10.1080/01616412.2017.1281195.

45. Baker SK, Chen ZL, Norris EH, Revenko AS, MacLeod AR, Strickland S. Bloodderived plasminogen drives brain inflammation and plaque deposition in a mouse model of Alzheimer's disease. Proc Natl Acad Sci U S A. 2018;115(41): E9687-e9696. https://doi.org/10.1073/pnas.1811172115.

46. Song F, Poljak A, Kochan NA, Raftery M, Brodaty H, Smythe GA, Sachdev PS Plasma protein profiling of mild cognitive impairment and Alzheimer's disease using iTRAQ quantitative proteomics. Proteome Sci. 2014;12(1):5. https://doi.org/10.1186/1477-5956-12-5.

47. Muenchhoff J, Poljak A, Thalamuthu A, Gupta VB, Chatterjee P, Raftery M, Masters CL, Morris JC, Bateman RJ, Fagan AM, Martins RN, Sachdev PS. Changes in the plasma proteome at asymptomatic and symptomatic stages of autosomal dominant Alzheimer's disease. Sci Rep. 2016;6(1):29078. https://doi.org/10.1038/srep29078.

48. Noguchi M, Sato T, Nagai K, Utagawa I, Suzuki I, Arito M, lizuka N, Suematsu N, Okamoto K, Kato T, Yamaguchi N, Kurokawa MS. Roles of serum fibrinogen alpha chain-derived peptides in Alzheimer's disease. Int J Geriatr Psychiatry. 2014;29(8):808-18. https://doi.org/10.1002/gps.4047.

49. Foote M, Zhou Y. 14-3-3 proteins in neurological disorders. Int J Biochem Mol Biol. 2012;3(2):152-64.

50. Boom A, Pochet $R$, Authelet $M$, Pradier $L$, Borghgraef $P$, van Leuven $F$, Heizmann CW, Brion JP. Astrocytic calcium/zinc binding protein S100A6 over expression in Alzheimer's disease and in PS1/APP transgenic mice models. Biochim Biophys Acta. 2004;1742(1-3):161-8. https://doi.org/10.101 6/j.bbamcr.2004.09.011.

51. Power JH, Asad S, Chataway TK, Chegini F, Manavis J, Temlett JA, et al. Peroxiredoxin 6 in human brain: molecular forms, cellular distribution and association with Alzheimer's disease pathology. Acta Neuropathol. 2008; 115(6):611-22. https://doi.org/10.1007/s00401-008-0373-3.

52. Duits FH, Brinkmalm G, Teunissen CE, Brinkmalm A, Scheltens $P$, van der Flier WM, et al. Synaptic proteins in CSF as potential novel biomarkers for prognosis in prodromal Alzheimer's disease. Alzheimers Res Ther. 2018;10(1): 5 .

53. Devanarayan P, Devanarayan V, Llano DA. Identification of a simple and novel cut-point based cerebrospinal fluid and MRI signature for predicting Alzheimer's disease progression that reinforces the 2018 NIA-AA research framework. J Alzheimers Dis. 2019;68(2):537-50. https://doi.org/10.3233/JA D-180905.

54. Janelidze S, Mattsson N, Stomrud E, Lindberg O, Palmqvist S, Zetterberg H, Blennow K, Hansson O. CSF biomarkers of neuroinflammation and cerebrovascular dysfunction in early Alzheimer disease. Neurology. 2018; 91(9):e867-77. https://doi.org/10.1212/WNL.0000000000006082.

55. Shang J, Yamashita T, Fukui Y, Song D, Li X, Zhai Y, et al. Different associations of plasma biomarkers in Alzheimer's disease, mild cognitive impairment, vascular dementia, and ischemic stroke. J Clin Neurol (Seoul, Korea). 2018;14(1):29-34.

56. Zemva J, Schubert M. The role of neuronal insulin/insulin-like growth factor1 signaling for the pathogenesis of Alzheimer's disease: possible therapeutic implications. CNS Neurol Disord Drug Targets. 2014;13(2):322-37. https://doi. org/10.2174/18715273113126660141.

57. Anvar NE, Saliminejad K, Ohadi M, Kamali K, Daneshmand P, Khorshid HR. Association between polymorphisms in Interleukin-16 gene and risk of lateonset Alzheimer's disease. J Neurol Sci. 2015;358(1-2):324-7. https://doi. org/10.1016/j.jns.2015.09.344.

58. Farkas M, Keskitalo S, Smith DEC, Bain N, Semmler A, Ineichen B, Smulders $Y$, Blom H, Kulic L, Linnebank M. Hyperhomocysteinemia in Alzheimer's disease: the hen and the egg? J Alzheimers Dis. 2013;33(4):1097-104. https://doi.org/10.3233/JAD-2012-121378.

59. Selley ML. A metabolic link between S-adenosylhomocysteine and polyunsaturated fatty acid metabolism in Alzheimer's disease. Neurobiol 
Aging. 2007;28(12):1834-9. https://doi.org/10.1016/j.neurobiolaging.2006.08. 003.

60. Czech C, Berndt P, Busch K, Schmitz O, Wiemer J, Most V, Hampel H, Kastler J, Senn H. Metabolite profiling of Alzheimer's disease cerebrospinal fluid. PLoS One. 2012;7(2):e31501. https://doi.org/10.1371/journal.pone.0031501.

61. Frenkel-Pinter M, Shmueli MD, Raz C, Yanku M, Zilberzwige S, Gazit E, Segal D. Interplay between protein glycosylation pathways in Alzheimer's disease. Sci Adv. 2017;3(9):e1601576. https://doi.org/10.1126/sciadv.1601576.

62. Lin $\mathrm{CH}$, Yang HT, Lane HY. D-glutamate, D-serine, and D-alanine differ in their roles in cognitive decline in patients with Alzheimer's disease or mild cognitive impairment. Pharmacology, biochemistry, and behavior 2019; 185: 172760.

63. Yin JX, Maalouf M, Han P, Zhao M, Gao M, Dharshaun T, Ryan C, Whitelegge J, Wu J, Eisenberg D, Reiman EM, Schweizer FE, Shi J. Ketones block amyloid entry and improve cognition in an Alzheimer's model. Neurobiol Aging. 2016;39:25-37. https://doi.org/10.1016/j.neurobiolaging.2015.11.018.

64. Orešič M, Hyötyläinen T, Herukka S-K, Sysi-Aho M, Mattila I, Seppänan-Laakso T, Julkunen V, Gopalacharyulu PV, Hallikainen M, Koikkalainen J, Kivipelto M, Helisalmi S, Lötjönen J, Soininen H. Metabolome in progression to Alzheimer's disease. Transl Psychiatry. 2011;1(12):e57. https://doi.org/10.103 8/tp.2011.55.

65. Zhou M, Haque RU, Dammer EB, Duong DM, Ping L, Johnson ECB, et al. Targeted mass spectrometry to quantify brain-derived cerebrospinal fluid biomarkers in Alzheimer's disease. Clin Proteom. 2020;17(1):1-14.

66. Mateo I, Llorca J, Infante J, Rodríquez-Rodríquez E, Berciano J, Combarros O. Gene-gene interaction between 14-3-3 zeta and butyrylcholinesterase modulates Alzheimer's disease risk. Eur J Neurol. 2008;15(3):219-22. https:/ doi.org/10.1111/j.1468-1331.2008.02059.x.

67. Bader JM, Geyer PE, Müller JB, Strauss MT, Koch M, Leypoldt F, Koertvelyessy P, Bittner D, Schipke CG, Incesoy El, Peters O, Deigendesch N, Simons M, Jensen MK, Zetterberg H, Mann M. Proteome profiling in cerebrospinal fluid reveals novel biomarkers of Alzheimer's disease. Mol Syst Biol. 2020;16(6): e9356. https://doi.org/10.15252/msb.20199356.

68. George C, Gontier G, Lacube P, François J-C, Holzenberger M, Aïd S. The Alzheimer's disease transcriptome mimics the neuroprotective signature of IGF-1 receptor-deficient neurons. Brain. 2017;140(7):2012-27. https://doi. org/10.1093/brain/awx132.

69. Deng Y, Li B, Liu F, labal K, Grundke-labal I, Brandt R, Gong CX. Regulation between O-GlcNAcylation and phosphorylation of neurofilament-M and their dysregulation in Alzheimer disease. FASEB J. 2008;22(1):138-45. https:// doi.org/10.1096/fj.07-8309com.

70. Cuchillo-Ibañez I, Balmaceda V, Mata-Balaguer T, Lopez-Font I, Sáez-Valero J. Reelin in Alzheimer's disease, increased levels but impaired signaling: when more is less. J Alzheimers Dis. 2016;52(2):403-16. https://doi.org/10.3233/JA D-151193.

71. Morgan AR, Touchard S, Leckey C, O'Hagan C, Nevado-Holgado AJ, Barkhof $F$, et al. Inflammatory biomarkers in Alzheimer's disease plasma. Alzheimers Dement. 2019;15(6):776-87. https://doi.org/10.1016/j.jalz.2019.03.007.

72. Stamate D, Kim M, Proitsi P, Westwood S, Baird A, Nevado-Holgado A, Hye A, Bos I, Vos SJB, Vandenberghe R, Teunissen CE, Kate MT, Scheltens P, Gabel S, Meersmans K, Blin O, Richardson J, de Roeck E, Engelborghs S, Sleegers K, Bordet R, Ramit L, Kettunen P, Tsolaki M, Verhey F, Alcolea D, Lléo A, Peyratout G, Tainta M, Johannsen P, Freund-Levi Y, Frölich L, Dobricic V, Frisoni GB, Molinuevo JL, Wallin A, Popp J, Martinez-Lage P, Bertram L, Blennow K, Zetterberg H, Streffer J, Visser PJ, Lovestone S, Legido-Quigley C. A metabolitebased machine learning approach to diagnose Alzheimer-type dementia in blood: Results from the European Medical Information Framework for Alzheimer disease biomarker discovery cohort. Alzheimers Dement (N Y). 2019; 5(1):933-8. https:/doi.org/10.1016/.jtrci.2019.11.001.

73. van Eyk JE, Snyder MP. Precision medicine: role of proteomics in changing clinical management and care. J Proteome Res. 2019;18(1):1-6. https://doi. org/10.1021/acs.jproteome.8b00504.

74. Markaki I, Bergström S, Tsitsi P, Remnestål J, Månberg A, Hertz E, Paslawski W, Sorjonen K, Uhlén M, Mangone G, Carvalho S, Rascol O, Meissner WG, Magnin E, Wüllner U, Corvol JC, Nilsson P, Svenningsson P. Cerebrospinal fluid levels of kininogen-1 indicate early cognitive impairment in Parkinson's disease. Mov Disord. 2020;35(11):2101-6. https://doi.org/10.1002/mds.28192.

75. Gaudet P, Livstone MS, Lewis SE, Thomas PD. Phylogenetic-based propagation of functional annotations within the Gene Ontology consortium. Brief Bioinform. 2011;12(5):449-62. https://doi.org/10.1093/bib/ bbr042.
76. Fejtova A, Davydova D, Bischof F, Lazarevic V, Altrock WD, Romorini S, Schöne C, Zuschratter W, Kreutz MR, Garner CC, Ziv NE, Gundelfinger ED. Dynein light chain regulates axonal trafficking and synaptic levels of Bassoon. J Cell Biol. 2009:185(2):341-55. https://doi.org/10.1083/jcb.200807155.

77. Meng X, McGraw CM, Wang W, Jing J, Yeh S-Y, Wang L, et al. Neurexophilin4 is a selectively expressed a-neurexin ligand that modulates specific cerebellar synapses and motor functions. Elife. 2019;8. https://doi. org/10.7554/eLife.46773.

78. Whiley L, Sen A, Heaton J, Proitsi P, García-Gómez D, Leung R, Smith N, Thambisetty M, Kloszewska I, Mecocci P, Soininen H, Tsolaki M, Vellas B, Lovestone S, Legido-Quigley C, AddNeuroMed Consortium. Evidence of altered phosphatidylcholine metabolism in Alzheimer's disease. Neurobiol Aging. 2014;35(2):271-8. https://doi.org/10.1016/j.neurobiolaging.2013.08.001.

79. VanGuilder HD, Farley JA, Yan H, van Kirk CA, Mitschelen M, Sonntag WE, et al. Hippocampal dysregulation of synaptic plasticity-associated proteins with age-related cognitive decline. Neurobiol Dis. 2011;43(1):201-12. https:// doi.org/10.1016/j.nbd.2011.03.012.

80. Jongbloed W, van Dijk KD, Mulder SD, van de Berg WDJ, Blankenstein MA, van der Flier W, Veerhuis R. Clusterin levels in plasma predict cognitive decline and progression to Alzheimer's disease. J Alzheimers Dis. 2015;46(4): 1103-10. https://doi.org/10.3233/JAD-150036.

81. Bettcher BM, Neuhaus J, Wynn MJ, Elahi FM, Casaletto KB, Saloner R, et al. Increases in a pro-inflammatory chemokine, MCP-1, are related to decreases in memory over time. Front Aging Neurosci. 2019;11:25.

82. Cortes-Canteli M, Zamolodchikov D, Ahn HJ, Strickland S, Norris EH. Fibrinogen and altered hemostasis in Alzheimer's disease. J Alzheimers Dis. 2012;32(3):599-608. https://doi.org/10.3233/JAD-2012-120820.

83. Catricala S, Torti M, Ricevuti G. Alzheimer disease and platelets: how's that relevant. Immun Ageing. 2012;9(1):20. https://doi.org/10.1186/1742-4933-9-20.

84. Wesenhagen KEJ, Teunissen CE, Visser PJ, Tijms BM. Cerebrospinal fluid proteomics and biological heterogeneity in Alzheimer's disease: a literature review. Crit Rev Clin Lab Sci. 2020;57(2):86-98.

85. Huynh MB, Ouidja MO, Chantepie S, Carpentier G, Maïza A, Zhang G, Vilares J, Raisman-Vozari R, Papy-Garcia D. Glycosaminoglycans from Alzheimer's disease hippocampus have altered capacities to bind and regulate growth factors activities and to bind tau. PLoS One. 2019;14(1):e0209573. https://doi. org/10.1371/journal.pone.0209573.

86. Ovsepian SV, O'Leary VB. Neuronal activity and amyloid plaque pathology: an update. J Alzheimers Dis. 2016;49(1):13-9. https://doi.org/10.3233/JAD-1 50544.

87. Peña F, Gutiérrez-Lerma A, Quiroz-Baez R, Arias C. The role of beta-amyloid protein in synaptic function: implications for Alzheimer's disease therapy. Curr Neuropharmacol. 2006;4(2):149-63. https://doi.org/10.2174/15701 5906776359531.

\section{Publisher's Note}

Springer Nature remains neutral with regard to jurisdictional claims in published maps and institutional affiliations.

Ready to submit your research? Choose BMC and benefit from:

- fast, convenient online submission

- thorough peer review by experienced researchers in your field

- rapid publication on acceptance

- support for research data, including large and complex data types

- gold Open Access which fosters wider collaboration and increased citations

- maximum visibility for your research: over $100 \mathrm{M}$ website views per year

At BMC, research is always in progress.

Learn more biomedcentral.com/submissions 